Supporting Information

\title{
Photochemical Creation of Covalent Organic 2D Monolayer Objects in Defined Shapes via a Lithographic 2D-Polymerization
}

Marco Servalli, Kemal Celebi, Payam Payamyar, Liqing Zheng, Miroslav Položij, Benjamin Lowe, Agnieszka Kuc, Tobias Schwarz, Kerstin Thorwarth, Andreas Borgschulte, Thomas Heine, Renato Zenobi, A. Dieter Schlüter 


\section{Table of contents}

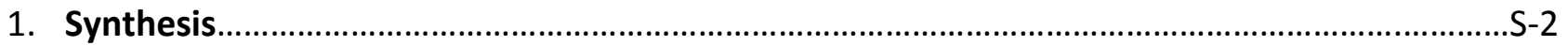

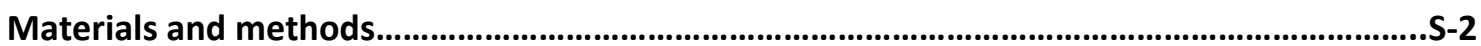

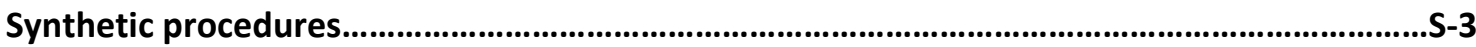

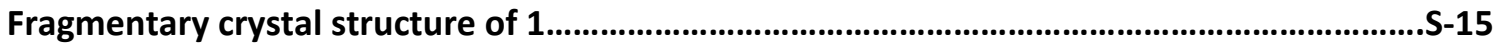

2. Preparation of Langmuir monolayers at the air/water interface.............................................S-16

Spreading conditions and preparation of the monolayer............................................................16

Transfer of the monolayer onto substrates.....................................................................................17

Packing considerations according to MMA values...........................................................................

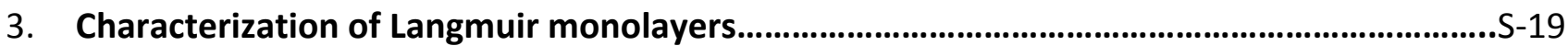

AFM height analysis of the monolayers and film transfer..........................................................

In-situ Brewster's angle microscopy.....................................................................................................

Visualization of the monolayers at the interface................................................................................S-21

UV/Vis absorption and emission spectra in solution and on quartz........................................S-22

In-situ fluorescence spectroscopy at the interface.......................................................................23

Polymerization at the air/water interface.....................................................................................

Tip-Enhanced Raman Spectroscopy (TERS) ......................................................................................

Scanning electron microscopy (SEM) ................................................................................................

X-ray Photoelectron Spectroscopy (XPS) .........................................................................................2-26

4. Optically-Rewritable Molecular Paper...........................................................................................28

Confocal Laser Scanning Microscopy (CLSM) ................................................................................2-28

Writing at the air/water interface with a $405 \mathrm{~nm}$ laser pointer..............................................

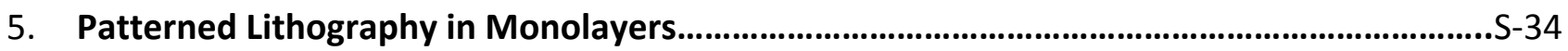

6. Molecular model for monomer and polymer monolayer.......................................................S-37

Monomer monolayer structure................................................................................................39

Polymer monolayer structure................................................................................................................41

7. Amphiphilicity and spreading of anthraphanes at the air/water interface.........................S-43

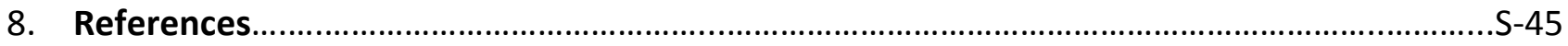




\section{Synthesis}

\section{Materials and methods}

All reactions were carried out under nitrogen by using standard Schlenk techniques and dry solvents unless otherwise noted. Dry diethyl ether, dry methanol, dry DMI and dry toluene were purchased over molecular sieves from Acros and used directly. Diisopropyl amine was dried by passing it over a column of activated neutral aluminum oxide (Brockmann Activity I, Sigma-Aldrich) according to the literature. ${ }^{1} \mathrm{Pd}\left(\mathrm{PPh}_{3}\right)_{4}$ catalyst was freshly prepared following the literature procedure ${ }^{2}$ and stored in a glove-box in the dark under $\mathrm{N}_{2}$ at room temperature. All reagents were purchased from Acros, Aldrich or $\mathrm{TCl}$, and used without further purification. Column chromatography for purification of the products was performed by using Merck silica gel Si60 (particle size 40-63 $\mu \mathrm{m}$ ).

NMR was recorded on a Bruker AVANCE $\left({ }^{1} \mathrm{H}: 300 \mathrm{MHz},{ }^{13} \mathrm{C}: 75 \mathrm{MHz}\right)$ at room temperature. The signal from the solvents was used as internal standard for chemical shift $\left({ }^{1} \mathrm{H}: \delta=7.26 \mathrm{ppm},{ }^{13} \mathrm{C}: \delta=77.16 \mathrm{ppm}\right.$ for chloroform, ${ }^{1} \mathrm{H}: \delta$ $=6.00 \mathrm{ppm},{ }^{13} \mathrm{C}: \delta=73.78 \mathrm{ppm}$ for 1,1,2,2-tetrachloroethane, ${ }^{19} \mathrm{~F}: \delta=-164.9 \mathrm{ppm}$ for hexafluorobenzene). When possible, proton and carbon signal assignments were performed with the help of 2D-NMR experiments such as COSY, HSQC and HMBC (spectra not shown).

High resolution mass spectroscopy (HRMS) analyses were performed by the MS-service of the Laboratory for Organic Chemistry at ETH Zurich with spectrometers (ESI- and MALDI-ICR-FTMS: IonSpec Ultima Instrument). Either 3hydroxypicolinic acid (3-HPA) or trans-2-[3-(4-tert-butylphenyl)-2-methyl-2-propenylidene]malononitrile (DCTB) were used as matrix. 
<smiles>Fc1c(I)c(F)c(I)c(F)c1I</smiles>

\section{1,3,5-Trifluoro-2,4,6-triiodobenzene (2)}

Periodic acid (5.13 g, $22.5 \mathrm{mmol}, 1.50 \mathrm{eq}$ ) was suspended in $35 \mathrm{~mL}$ sulphuric acid at $0^{\circ} \mathrm{C}$. Finely ground potassium iodide (11.2 g, $67.6 \mathrm{mmol}, 4.50 \mathrm{eq})$ was added in portions over $5 \mathrm{~min}$ during which time iodine vapors evolved. 1,3,5Trifluorobenzene $(1.56 \mathrm{~mL}, 15.1 \mathrm{mmol}, 1.00 \mathrm{eq})$ was then added by syringe at $0^{\circ} \mathrm{C}$ and the reaction mixture was then heated to $70^{\circ} \mathrm{C}$ for $5 \mathrm{~h}$, during which time additional sulphuric acid can be added for better stirring. After cooling to room temperature, the reaction mixture was poured into $350 \mathrm{~g}$ crushed ice. $200 \mathrm{~mL}$ diethyl ether were added and the layers separated. The organic layer was washed once with $150 \mathrm{~mL}$ of a $15 \%$ solution of sodium thiosulfate, once with $150 \mathrm{~mL}$ water, dried over $\mathrm{MgSO}_{4}$ and concentrated to dryness. The residue was purified by sublimation: the temperature was kept at $50^{\circ} \mathrm{C}(p=0.04 \mathrm{mbar})$ for $1 \mathrm{~h}$, during which time a brown layer coated the cold finger, then it was increased to $110^{\circ} \mathrm{C}$ to allow sublimation of the product. Compound $\mathbf{2}$ was obtained as a white crystalline solid (7.60 g, $14.9 \mathrm{mmol}, 97 \%) . \mathrm{R}_{\mathrm{f}}$ (hexane): 0.6, $\mathrm{Mp}: 156-158^{\circ} \mathrm{C}$.

${ }^{13} \mathrm{C}-\mathrm{NMR}\left(75.5 \mathrm{MHz}, \mathrm{CDCl}_{3}\right.$ ) $\delta / \mathrm{ppm}: 162.4(\mathrm{dt}, J=243.5,7.8 \mathrm{~Hz}), 63.9$ (ddd, $J=34.9,34.1,3.9 \mathrm{~Hz}$ ).

${ }^{19} \mathrm{~F}-\mathrm{NMR}\left(282.5 \mathrm{MHz}, \mathrm{CDCl}_{3}\right)$ $) / \mathrm{ppm}: 68.83$.

HRMS (FT-MALDI): m/z calcd for $\mathrm{C}_{14} \mathrm{H}_{13} \mathrm{O}_{2}$ [M-H] $]^{+}$: 213.0910; found: 213.0909 . 


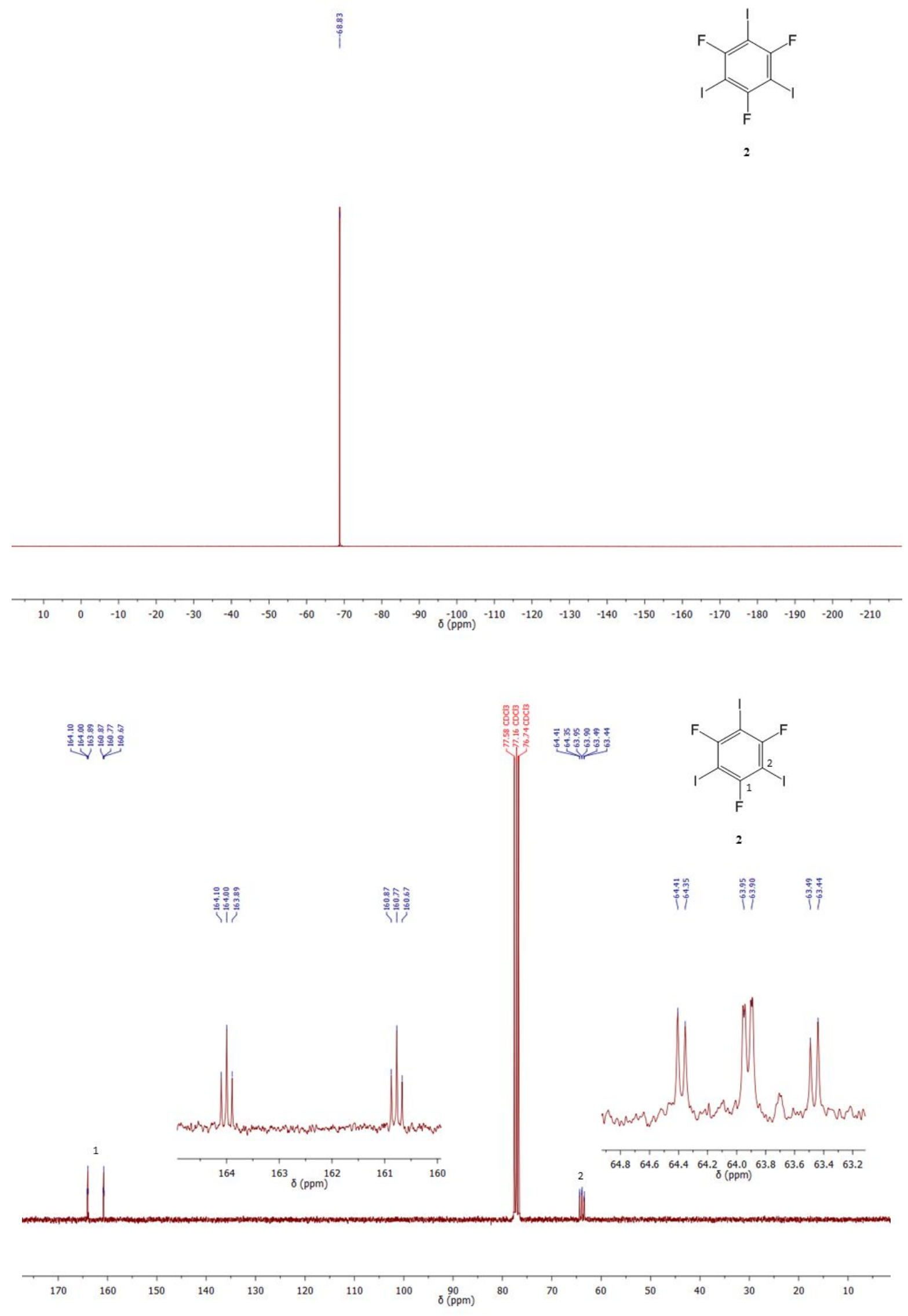

Figure S1. ${ }^{19} \mathrm{~F}$ - and ${ }^{13} \mathrm{C}-\mathrm{NMR}$ spectra of compound $\mathbf{2}$ in $\mathrm{CDCl}_{3}$. 


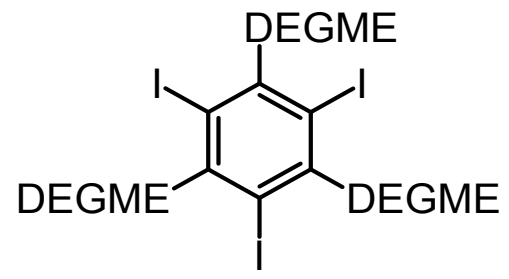

\section{1,3,5-Triiodo-2,4,6-tris(2-(2-methoxyethoxy)ethoxy)benzene (3)}

Sodium hydride ( $0.94 \mathrm{~g}, 23.5 \mathrm{mmol}, 6 \mathrm{eq}, 60 \%$ dispersion in mineral oil) was suspended in $8 \mathrm{~mL}$ dry diethyl ether in a $100 \mathrm{~mL}$ Schlenk flask. Dry 2-(2-methoxyethoxy)ethanol $(2.77 \mathrm{~mL}, 23.5 \mathrm{mmol}, 6 \mathrm{eq})$ diluted in $8 \mathrm{~mL}$ dry diethyl ether was slowly added to the hydride under vigorous stirring. The reaction mixture was then stirred at room temperature for $1 \mathrm{~h}$ until no more hydrogen evolution was detectable. The volatiles were removed by a stream of nitrogen and the residue was dried on $\mathrm{HV}$ for $15 \mathrm{~min} .15 \mathrm{~mL}$ dry DMI were then added to the jelly-like residue and to the resulting suspension, 1,3,5-trifluoro-2,4,6-triiodobenzene 2 ( $2.00 \mathrm{~g}, 3.92 \mathrm{mmol}, 1 \mathrm{eq})$ was added in small portions over $30 \mathrm{~min}$ under vigorous stirring. The obtained orange suspension was stirred overnight at room temperature and then poured into $80 \mathrm{~mL}$ saturated $\mathrm{NaHCO}_{3}$ solution. The white precipitate was collected by filtration and washed with water until the $\mathrm{pH}$ of the filtrate resulted neutral. Purification by flash column chromatography (70\% EtOAc in hexane) afforded compound 3 as a white waxy solid (2.89 g, $3.57 \mathrm{mmol}, 91 \%) . \mathrm{R}_{\mathrm{f}}: 0.20, \mathrm{Mp}: 57-60^{\circ} \mathrm{C}$.

${ }^{1} \mathrm{H}-\mathrm{NMR}\left(300 \mathrm{MHz}, \mathrm{CDCl}_{3}\right)$ 8/ppm: $4.17(\mathrm{t}, J=5.2 \mathrm{~Hz}, 6 \mathrm{H}), 4.00(\mathrm{t}, J=5.2 \mathrm{~Hz}, 6 \mathrm{H}), 3.85-3.75(\mathrm{~m}, 6 \mathrm{H}), 3.65-3.56(\mathrm{~m}$, $6 \mathrm{H}), 3.41(\mathrm{~s}, 9 \mathrm{H})$.

${ }^{13} \mathrm{C}-\mathrm{NMR}\left(75.5 \mathrm{MHz}, \mathrm{CDCl}_{3}\right)$ 8/ppm: 160.3, 83.5, 72.4, 72.2, 71.0, 70.1, 59.3.

HRMS (FT-MALDI): $\mathrm{m} / \mathrm{z}$ calcd for $\mathrm{C}_{21} \mathrm{H}_{33} \mathrm{l}_{3} \mathrm{O}_{9}[\mathrm{M}]^{+}:$: 809.9253; found: 809.9253 . 


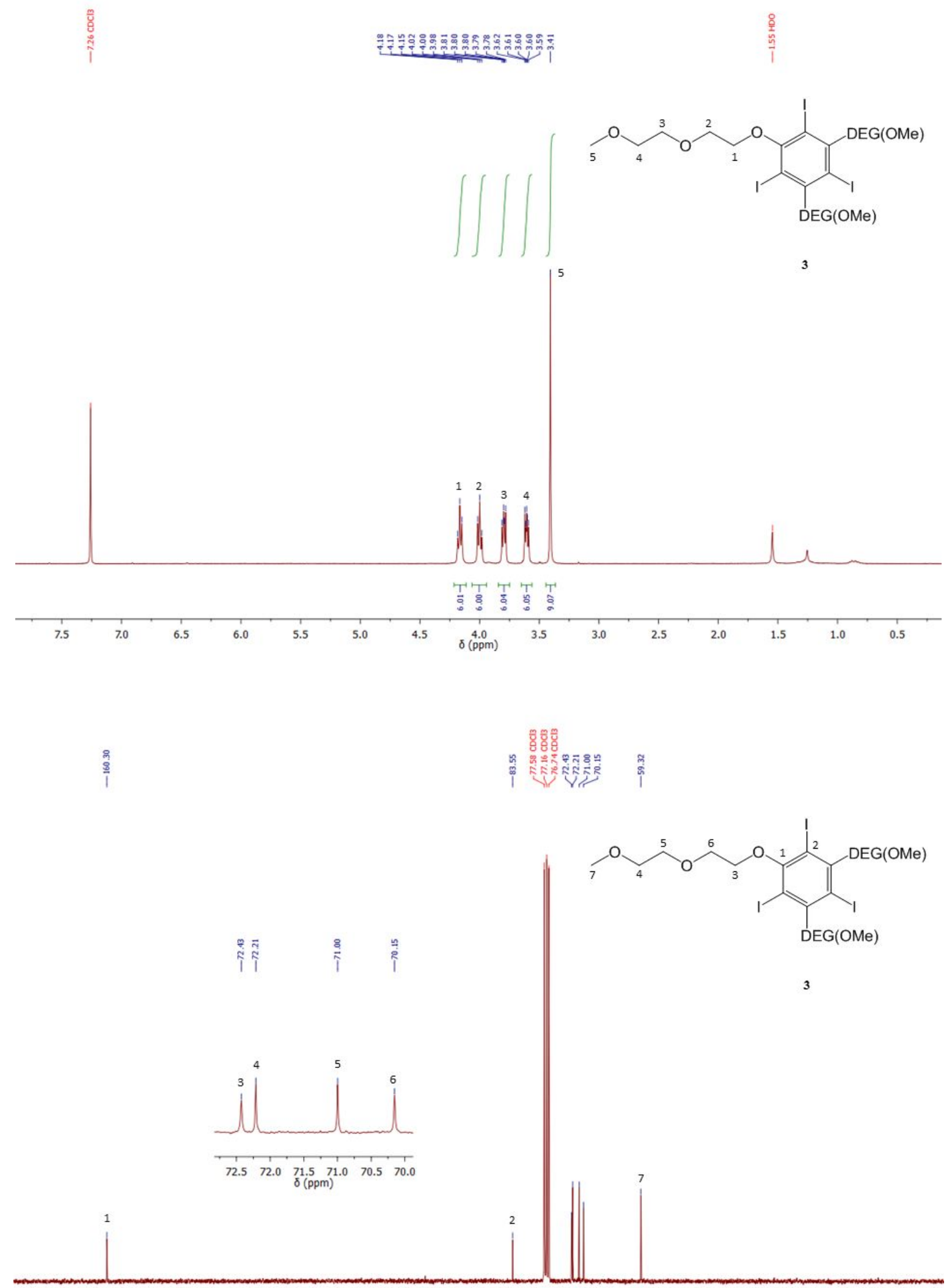

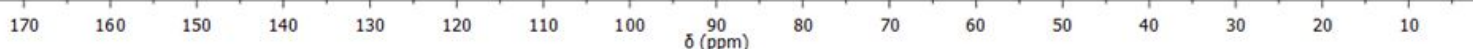

Figure S2. ${ }^{1} \mathrm{H}$ - and ${ }^{13} \mathrm{C}-\mathrm{NMR}$ spectra of compound $\mathbf{3}$ in $\mathrm{CDCl}_{3}$. 


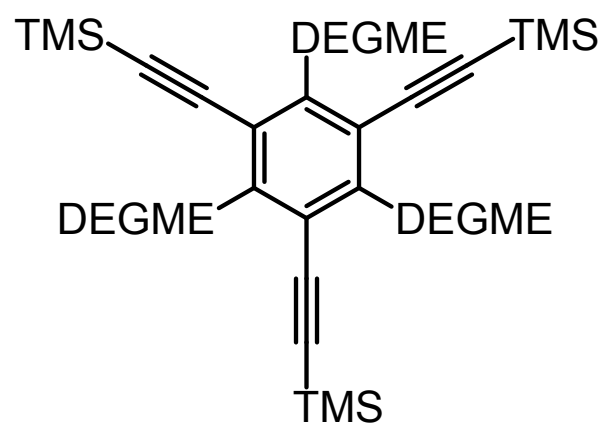

\section{((2,4,6-Tris(2-(2-methoxyethoxy)ethoxy)benzene-1,3,5-triyl)tris(ethyne-2,1-diyl))tris(trimethylsilane) (4)}

Compound $3(1.00 \mathrm{~g}, 1.23 \mathrm{mmol}, 1.00 \mathrm{eq})$ was placed in a dry $50 \mathrm{~mL}$ Schlenk tube along with catalyst $\mathrm{Pd}(\mathrm{OAc})_{2}(14.0$ $\mathrm{mg}, 0.06 \mathrm{mmol}, 0.05 \mathrm{eq})$, co-catalyst Cul $(11.0 \mathrm{mg}, 0.06 \mathrm{mmol}, 0.05 \mathrm{eq})$ and ligand tri-tert-butylphosphonium tetrafluoroborate $(36.0 \mathrm{mg}, 0.12 \mathrm{mmol}, 0.10 \mathrm{eq})$. In a separate Schlenk tube, $15 \mathrm{~mL}$ dry diisopropylamine were degassed by four cycles of freeze-pump-thaw and then added by syringe to the reactants. Trimethylsilylacetylene $(0.90 \mathrm{~mL}, 6.17 \mathrm{mmol}, 5 \mathrm{eq})$ was added and the reaction mixture was sealed and heated to $65^{\circ} \mathrm{C}$ for $48 \mathrm{~h}$ under an argon atmosphere. Formation of a beige precipitate indicated the start of the reaction. After cooling to room temperature, the reaction mixture was filtered through a celite pad and concentrated to dryness. Separation by column chromatography (30\% EtOAc in hexane) afforded the title compound as yellow oil (641 mg, $0.89 \mathrm{mmol}, 72 \%$ ). $\mathrm{R}_{\mathrm{f}}: 0.36$.

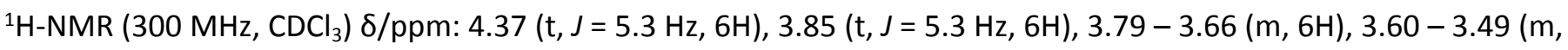
$6 \mathrm{H}), 3.37(\mathrm{~s}, 9 \mathrm{H}), 0.23(\mathrm{~s}, 27 \mathrm{H})$.

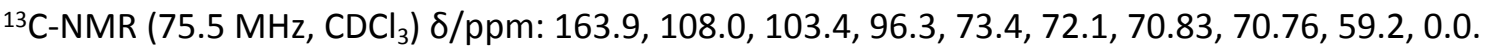

HRMS (FT-MALDI): m/z calcd for $\mathrm{C}_{36} \mathrm{H}_{60} \mathrm{O}_{9} \mathrm{Si}_{3}[\mathrm{M}]^{+}:$: 720.3540; found: 720.3539 . 


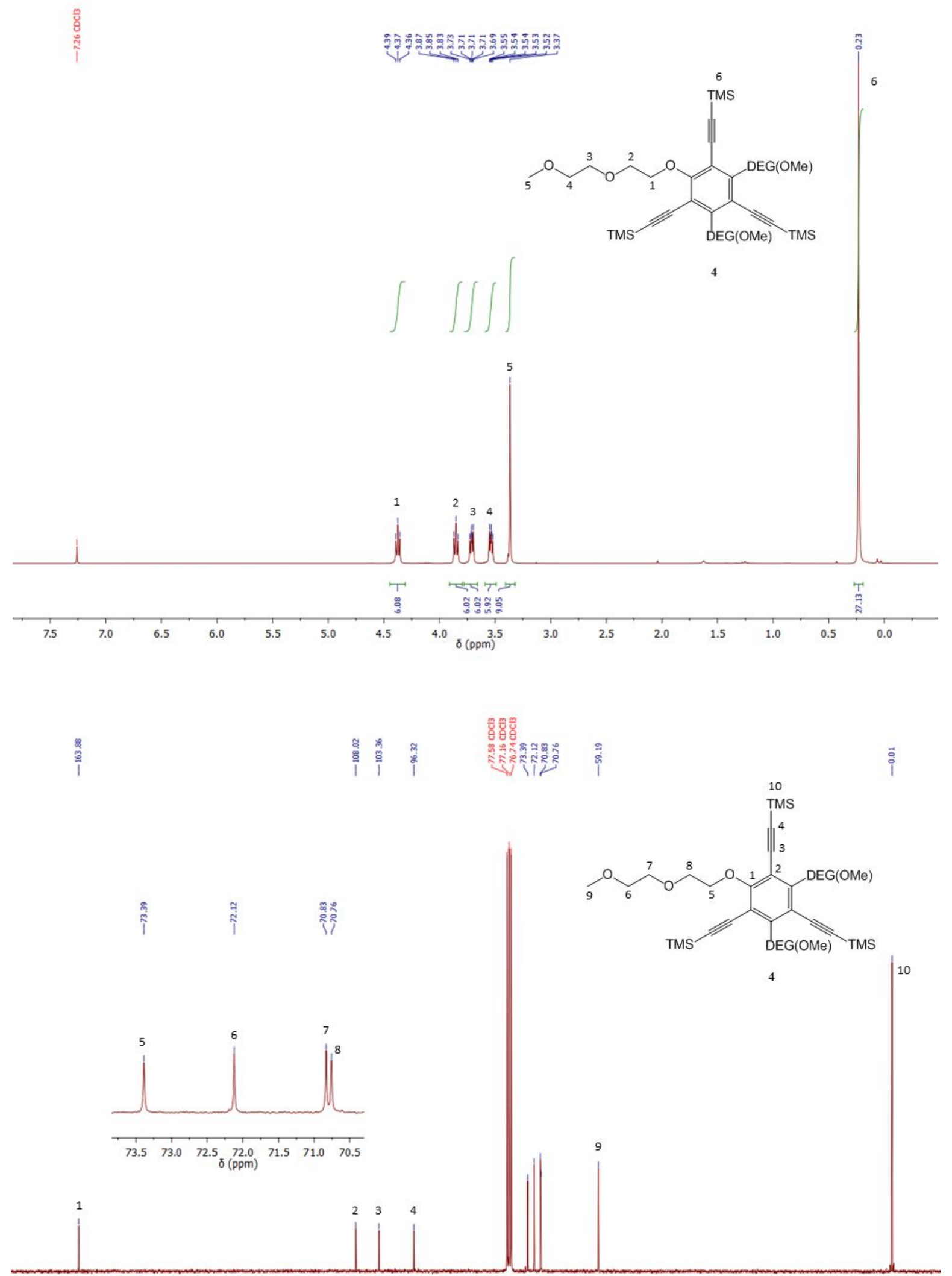

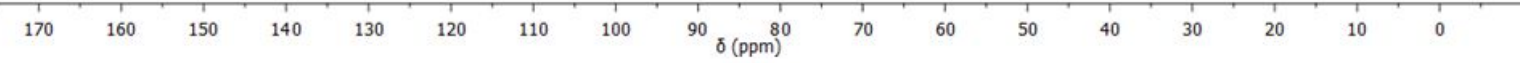

Figure S3. ${ }^{1} \mathrm{H}$ - and ${ }^{13} \mathrm{C}-\mathrm{NMR}$ spectra of compound 4 in $\mathrm{CDCl}_{3}$. 


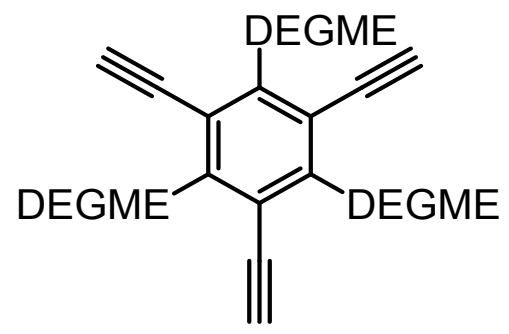

\section{1,3,5-triethynyl-2,4,6-tris(2-(2-methoxyethoxy)ethoxy)benzene (5)}

Compound 4 ( $0.54 \mathrm{~g}, 0.75 \mathrm{mmol}, 1 \mathrm{eq}$ ) was dissolved in a solvent mixture of $5 \mathrm{~mL}$ THF and $2 \mathrm{~mL} \mathrm{MeOH}$. Potassium carbonate $\left(13.0 \mathrm{mg}, 0.09 \mathrm{mmol}, 0.12 \mathrm{eq}\right.$ ) and $0.5 \mathrm{~mL} \mathrm{H} \mathrm{H}_{2} \mathrm{O}$ were added and the reaction mixture was stirred at room temperature for 16 h. $10 \mathrm{~mL} \mathrm{H} \mathrm{H}_{2} \mathrm{O}$ were added to the reaction mixture and the organics were removed in vacuo. The aqueous residue was subsequently extracted with diethyl ether $(3 \times 20 \mathrm{~mL})$ and the combined organic phases were dried over $\mathrm{MgSO}_{4}$ and concentrated to dryness. Purification by flash column chromatography (EtOAc) afforded the title compound 5 as a yellow oil (341 mg, $0.67 \mathrm{mmol}, 90 \%) . \mathrm{R}_{\mathrm{f}}: 0.36$.

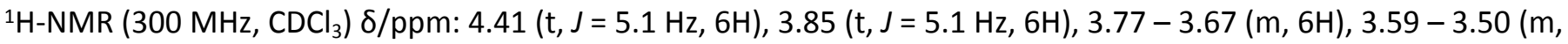
$6 \mathrm{H}), 3.43(\mathrm{~s}, 3 \mathrm{H}), 3.38(\mathrm{~s}, 9 \mathrm{H})$.

${ }^{13} \mathrm{C}-\mathrm{NMR}\left(75.5 \mathrm{MHz}, \mathrm{CDCl}_{3}\right.$ ) $\delta / \mathrm{ppm}: 164.5,107.3,86.1,75.0,73.5,72.0,70.7,70.4,59.1$.

HRMS (FT-MALDI): $\mathrm{m} / \mathrm{z}$ calcd for $\mathrm{C}_{27} \mathrm{H}_{36} \mathrm{NaO}_{9}[\mathrm{M}-\mathrm{Na}]^{+}:$527.2252; found: 527.2250 . 


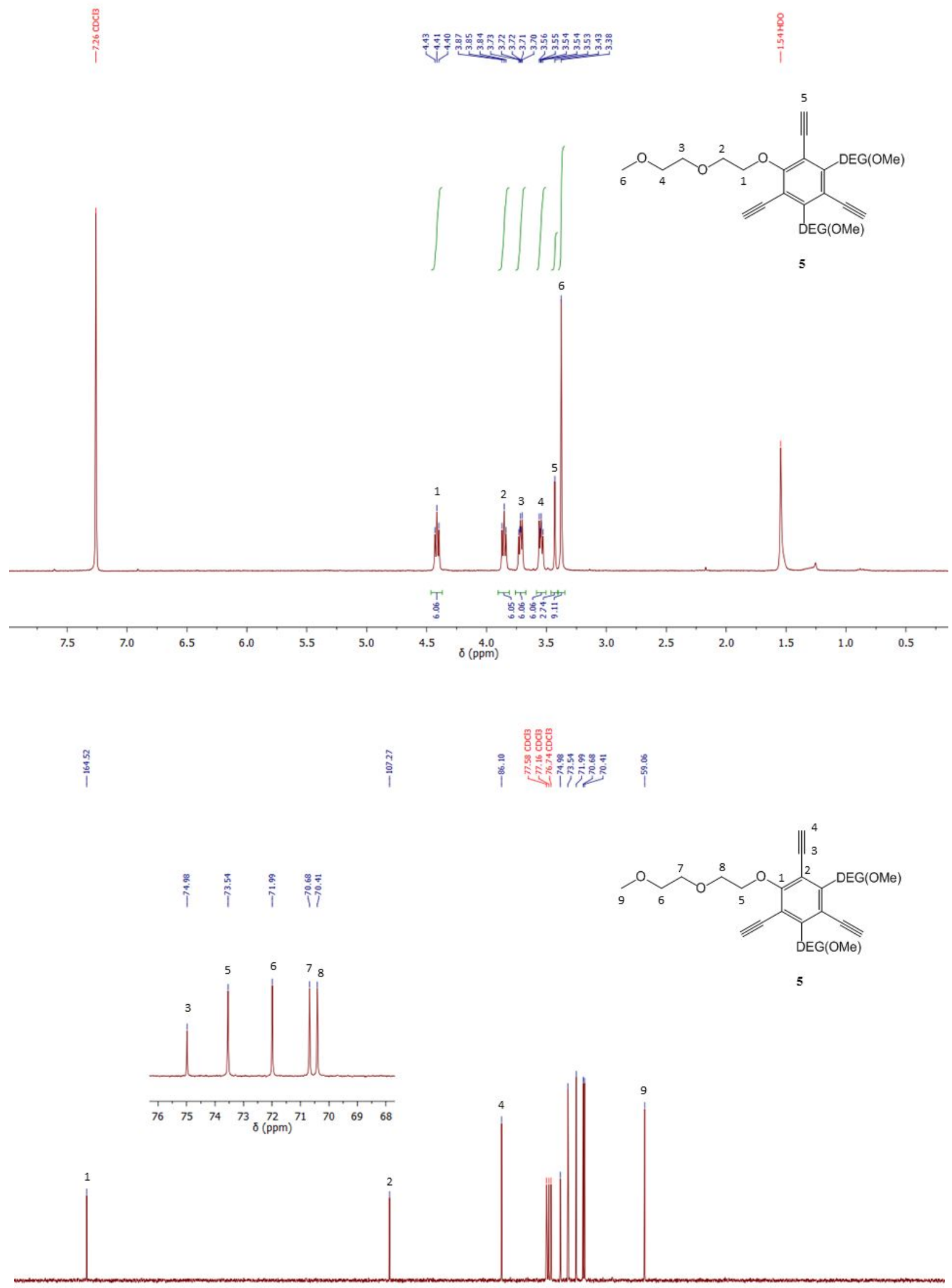

\begin{tabular}{|c|c|c|c|c|c|c|c|c|c|c|c|c|c|c|c|}
\hline 170 & 160 & 150 & 140 & 130 & 120 & 110 & 100 & $\stackrel{90}{90}$ & 80 & 70 & 60 & 50 & 40 & 30 & 20 \\
\hline
\end{tabular}

Figure S4. ${ }^{1} \mathrm{H}$ - and ${ }^{13} \mathrm{C}-\mathrm{NMR}$ spectra of compound $\mathbf{5}$ in $\mathrm{CDCl}_{3}$. 


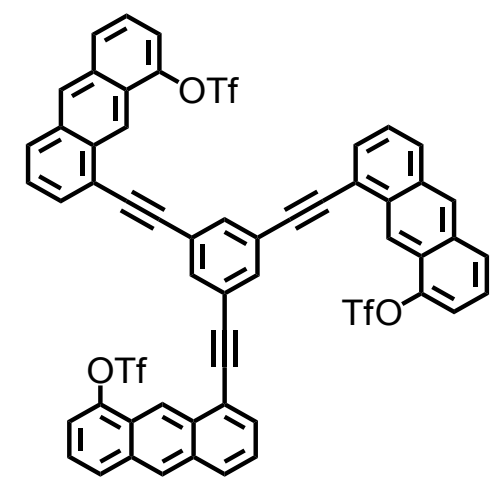

\section{Anthraphane precursor (6)}

(benzene-1,3,5-triyltris(ethyne-2,1-diyl))tris(anthracene-8,1-diyl) tris(trifluoromethanesulfonate)

Compound 6 was synthesised according to the literature procedure. ${ }^{3}$

${ }^{1} \mathrm{H}-\mathrm{NMR}\left(300 \mathrm{MHz}, \mathrm{CD}_{2} \mathrm{Cl}_{4}\right)$ 8/ppm: $9.36(\mathrm{~s}, 1 \mathrm{H}), 8.61(\mathrm{~s}, 1 \mathrm{H}), 8.19(\mathrm{~s}, 1 \mathrm{H}), 8.16-8.08(\mathrm{~m}, 2 \mathrm{H}), 7.98(\mathrm{~d}, J=7.1 \mathrm{~Hz}, 1 \mathrm{H})$, $7.63(\mathrm{dd}, J=8.6 \mathrm{~Hz}, 7.0 \mathrm{~Hz}, 1 \mathrm{H}), 7.58-7.50(\mathrm{~m}, 2 \mathrm{H})$.

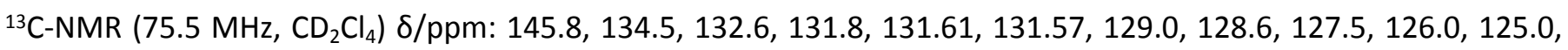
$124.3,124.0,121.3,118.9,118.7\left(q, J_{\mathrm{CF}}=321.0 \mathrm{~Hz}\right), 117.3,94.0,88.0$.

${ }^{19} \mathrm{~F}-\mathrm{NMR}\left(282.5 \mathrm{MHz}, \mathrm{CD}_{2} \mathrm{Cl}_{4}\right)$ 8/ppm: -76.65.

HRMS (FT-MALDI): $\mathrm{m} / \mathrm{z}$ calcd for $\mathrm{C}_{57} \mathrm{H}_{27} \mathrm{~F}_{9} \mathrm{O}_{9} \mathrm{~S}_{3}[\mathrm{M}]^{+}:$: 1122.0668; found: 1122.0673 . 


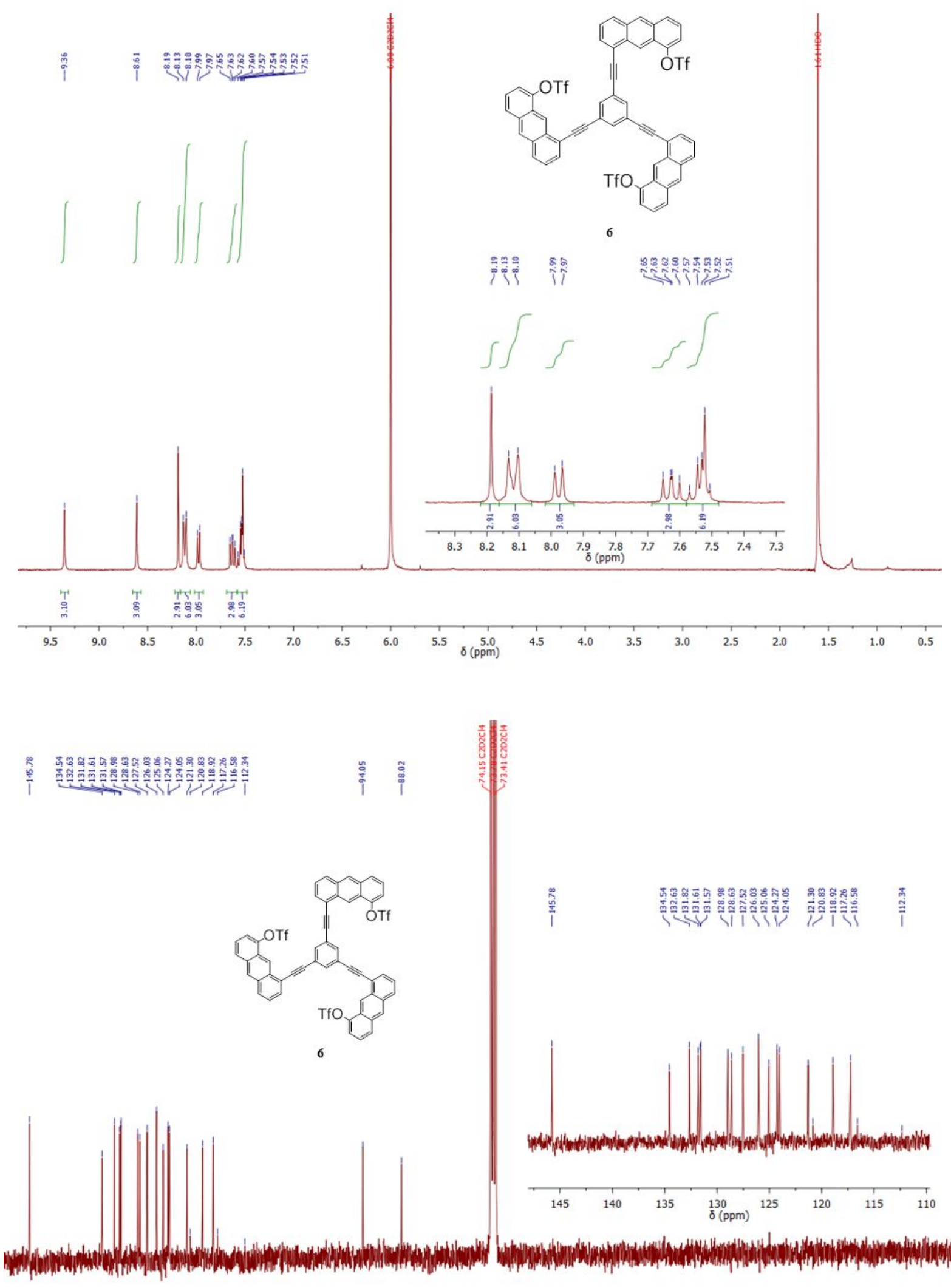

$\begin{array}{lllllllllllllllllllllllllllllllllllllllllllll}145 & 140 & 135 & 130 & 125 & 120 & 115 & 110 & 105 & 100 & 95 & 90 & 85 & 80 & 75 & 70 & 65 & 60 & 55 & 50 & 45 & 40 & 35 & 30 & 25 & 20 & 15 & 10 & 5\end{array}$

Figure S5. ${ }^{1} \mathrm{H}$ - and ${ }^{13} \mathrm{C}-\mathrm{NMR}$ spectra of compound 6 in $\mathrm{CDCl}_{3}$. 


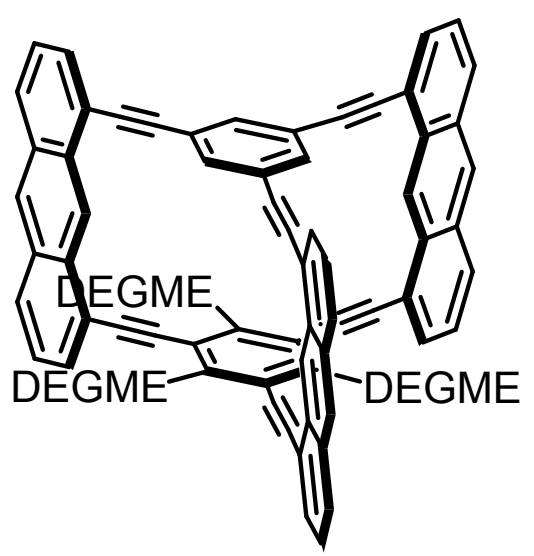

\section{Anthraphane-tri(DEGME) (1)}

Precursor 6 (500 mg, $0.44 \mathrm{mmol}, 1.00 \mathrm{eq}$ ) was suspended in $222 \mathrm{~mL}$ dry toluene (2.00 mM) with compound 5 (225 $\mathrm{mg}, 0.44 \mathrm{mmol}, 1.00 \mathrm{eq})$ and dry triethylamine $(12.4 \mathrm{~mL}, 89.0 \mathrm{mmol}, 200 \mathrm{eq})$. The reaction mixture was degassed by cooling it to $-80^{\circ} \mathrm{C}$ with an acetone-dry ice bath and then performing five cycles of vacuum (10 min) and nitrogen backfilling. $\mathrm{Pd}\left(\mathrm{PPh}_{3}\right)_{4}(154 \mathrm{mg}, 0.13 \mathrm{mmol}, 0.30 \mathrm{eq})$ was added with $\mathrm{N}_{2}$ counter-flow and the suspension was degassed twice again and backfilled with argon after the last cycle. After warming to room temperature, the reaction mixture was put in a preheated bath at $80^{\circ} \mathrm{C}$ and stirred in the dark under argon for $5 \mathrm{~d}$. After cooling to room temperature, the reaction mixture was filtered and the filtrate was concentrated to dryness. The brownish residue was washed with $\mathrm{MeOH}$ to obtain a beige solid, which was collected by filtration and rinsed with more $\mathrm{MeOH}$ until the filtrate resulted colorless. The crude product was then purified by column chromatography (1\% $\mathrm{MeOH}$ in $\mathrm{DCM}$ ) and the obtained solid was recrystallized from boiling THF to obtain 1 as pale yellow needles ( $217 \mathrm{mg}, 0.16 \mathrm{mmol}, 42 \%)$. $\mathrm{R}_{\mathrm{f}}$ : 0.67 (blue fluorescence with $\lambda=366 \mathrm{~nm}$ ), $\mathrm{Mp}$ : decomposes above $280^{\circ} \mathrm{C}$.

${ }^{1} \mathrm{H}-\mathrm{NMR}\left(300 \mathrm{MHz}, \mathrm{CDCl}_{3}\right.$ ) 8/ppm: $9.49(\mathrm{~s}, 3 \mathrm{H}), 8.50$ (s, 3H), 8.06 (dd, J = 8.5, 3.9 Hz, 9H), 7.82-7.73 (m, 6H), 7.50 (dd, $J=8.4,7.1 \mathrm{~Hz}, 6 \mathrm{H}), 4.54(\mathrm{t}, J=5.1 \mathrm{~Hz}, 6 \mathrm{H}), 3.75(\mathrm{t}, J=5.1 \mathrm{~Hz}, 6 \mathrm{H}), 3.26(\mathrm{dd}, J=5.5,4.1 \mathrm{~Hz}, 6 \mathrm{H}), 2.86-2.70(\mathrm{~m}, 15 \mathrm{H})$.

${ }^{13} \mathrm{C}-\mathrm{NMR}\left(75.5 \mathrm{MHz}, \mathrm{CD}_{2} \mathrm{Cl}_{4}\right.$ ) 8/ppm: 163.2, 134.1, 131.5, 131.25, 131.22, 131.7, 130.3, 130.2, 129.4, 129.2, 127.5, $125.3,125.2,123.7,123.6,120.8,120.2$, 107.9, 95.1, 91.9, 88.4, 85.4, 70.9, 69.8, 69.5, 58.2.

HRMS (FT-MALDI): $\mathrm{m} / \mathrm{z}$ calcd for $\mathrm{C}_{81} \mathrm{H}_{60} \mathrm{O}_{9}$ [M] ${ }^{+}:$1176.4232; found: 1176.4224 . 

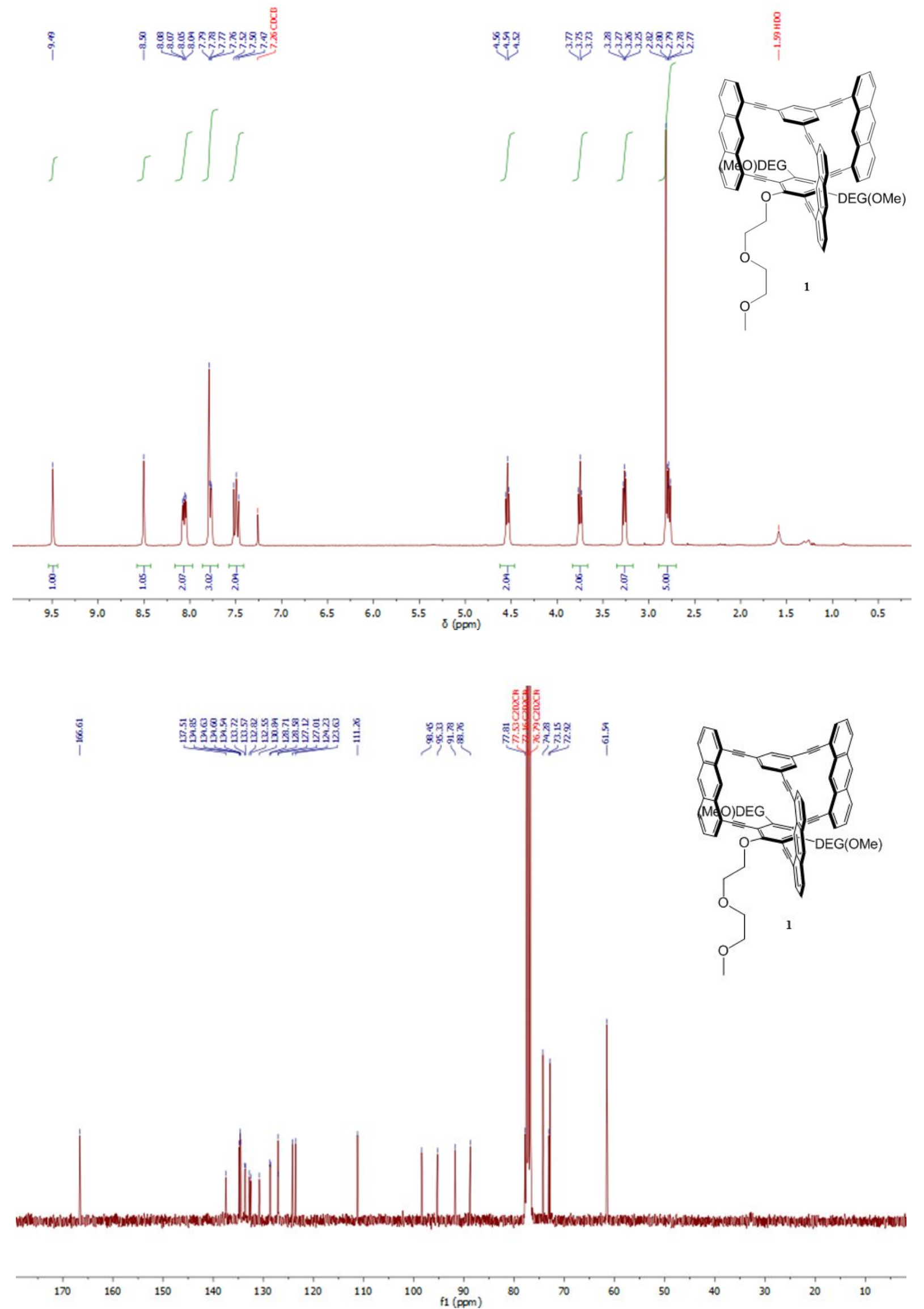

Figure S6. ${ }^{1} \mathrm{H}$ - and ${ }^{13} \mathrm{C}-\mathrm{NMR}$ spectra of compound $\mathbf{1}$ in $\mathrm{CDCl}_{3}$. 


\section{Fragmentary crystal structure of 1}

Crystals of 1 could be obtained from a variety of solvents (chloroform, THF, DMSO, toluene, nitrobenzene and odichlorobenzene) always in the form of yellow needles. However the quality of the crystals was always poor not allowing to obtain a fully resolved crystal structure. Disorder in the orientation of the main molecule coupled with additional disorder in the flexible DEGME chains were responsible for the observed diffraction. It was however possible to extract a model based on the fragmentary crystal structure solution, containing interesting information on the packing motif of 1 .

1 packed in layers and within the layers all the anthracene units were in an edge-to-face (etf) relationship (light blue in Figure S7). This kind of packing was commonly observed also for a hydrocarbon anthraphane. ${ }^{4}$ Interestingly enough, the DEGME chains prefer to fold back and interact with the main molecule skeleton rather than being fully extended and interpenetrating the layers.
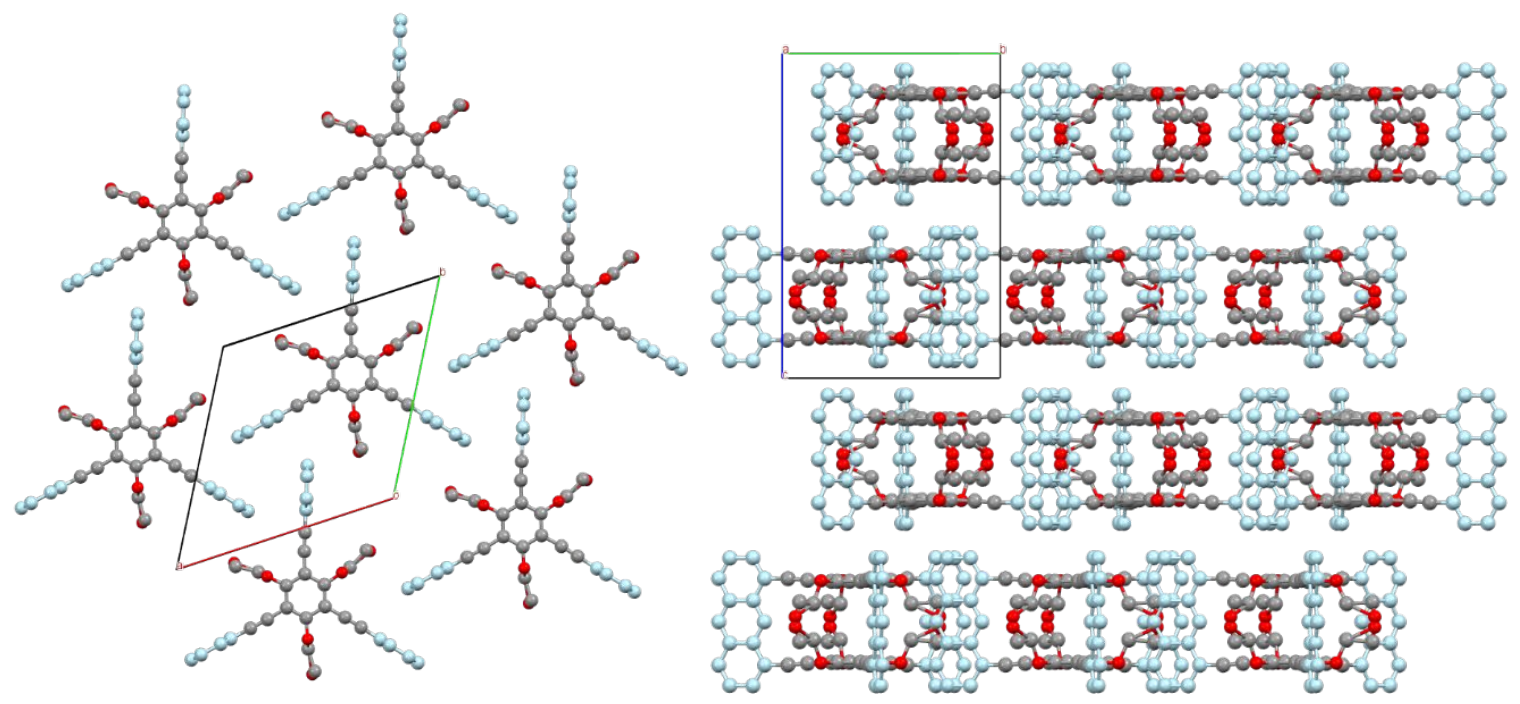

Figure S7. Packing motif based on a fragmentary structure solution due to excessive disorder in the crystals. This is not a real crystal structure! The anthracene units are displayed in light blue and are interacting with each other in an edge-to-face fashion. The molecules pack in layers and the DEGME chains are folding back towards the molecule's skeleton.

Crystals were measured on a Rigaku Oxford Diffraction XtaLAB Synergy-S Dualflex kappa diffractometer equipped with a Dectris Pilatus 300 HPAD detector and using microfocus sealed tube Cu-Ka radiation with mirror optics $(\lambda=$ $1.54178 \AA$ A). Data were integrated using CrysAlisPro and corrected for absorption effects using a combination of empirical (ABSPACK) and numerical corrections. ${ }^{5}$ The structures were solved using SHELXT ${ }^{6}$ and refined by full-matrix least-squares analysis $(\mathrm{SHELXL})^{6}$ using the program package OLEX2.7 


\section{Preparation of Langmuir monolayers at the air/water interface}

\section{Spreading conditions and preparation of the monolayer}

A KSV 2000 System 2 Langmuir trough equipped with a platinum Wilhelmy plate and dipper was used for this study. The trough is made of Teflon ${ }^{\circledR}$ and the barriers are of hydrophilic Delrin ${ }^{\circledR}$ (Figure S8). As subphase, Millipore water was used. For cleaning, the trough was first rinsed with Millipore water followed by chloroform, ethanol and chloroform again by wiping it with dust-free paper to remove all traces of organic material. The barriers were cleaned and wiped with ethanol and Millipore water. The trough was then filled with Millipore water and its surface treated by vacuum-suction to remove residual particles of dirt (cleanliness of the water surface was checked by UV lamp for residual fluorescence and by Brewster's angle microscopy). The Wilhelmy plate was rinsed with water and ethanol and then heated for a few seconds on a burner to incandescence.

For spreading of 1 , a stock solution $(0.25 \mathrm{mg} / \mathrm{mL})$ of a 1:1 solvent mixture of chloroform/cyclohexane was prepared. The solution was spread dropwise at the interface with the use of an air-tight glass microsyringe. Typical applied volumes were $200 \mu \mathrm{L}$. The solution was stored in a glass vial in the dark. If necessary, compression with the barriers was started after $30 \mathrm{~min}$ from the spreading to allow complete evaporation of chloroform. The compression rate was $3 \mathrm{~mm} / \mathrm{min}$.

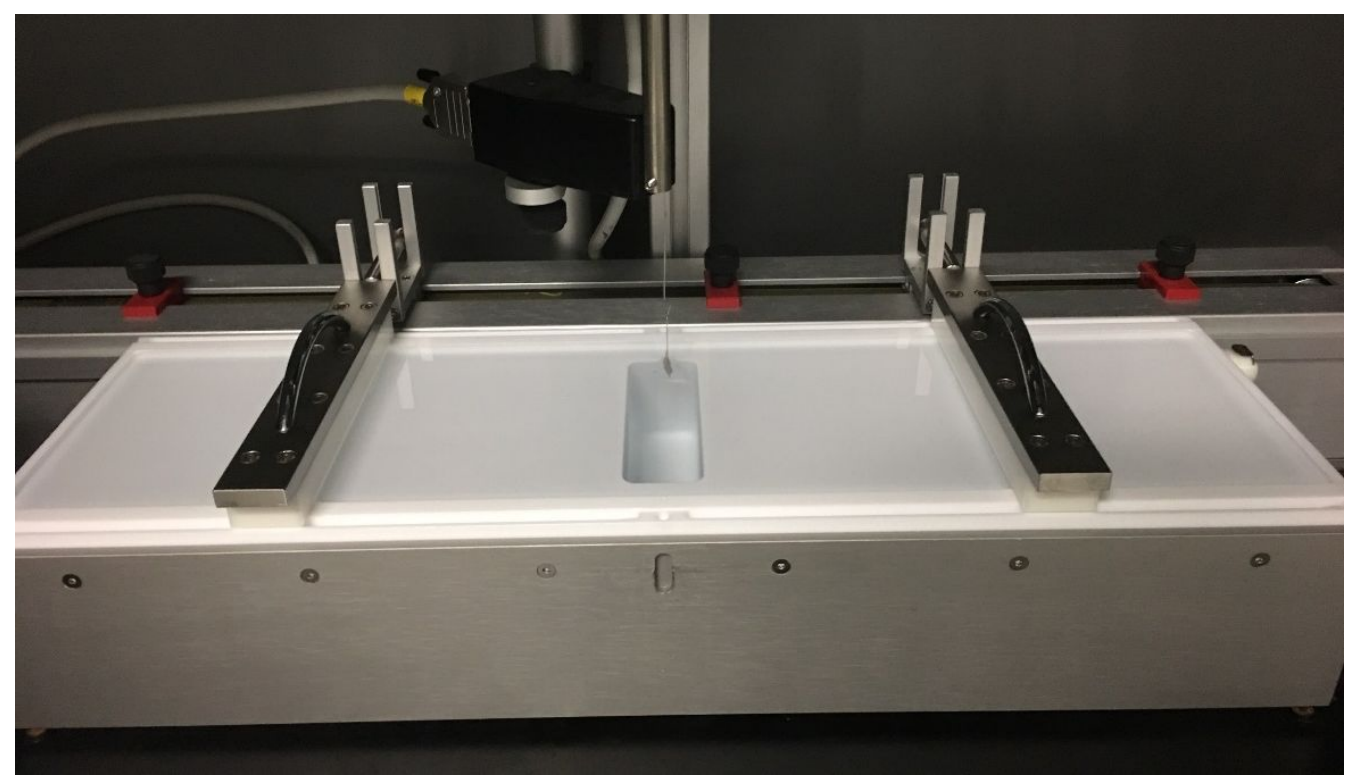

Figure S8. Langmuir trough used in this work. 


\section{Transfer of the monolayer onto substrates}

The Langmuir monolayers were transferred horizontally at constant surface pressure using a modified LangmuirSchaefer-technique (Figure S9): a tilted stage with the substrate was attached to the dipper and immersed just below the water surface; after spreading, compressing and reaching the target surface pressure, the stage was slowly pulled out of the water (transfer rate $0.5 \mathrm{~mm} / \mathrm{min}$ ) and the substrate left to dry overnight. The substrates used were: silicon oxide (285 nm) on silicon for AFM, template stripped gold for TERS and gold on silicon oxide for XPS.

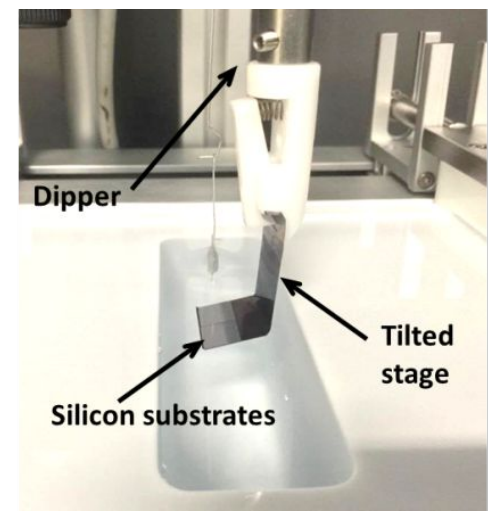

1) Lifting of substrates

2) Drying overnight

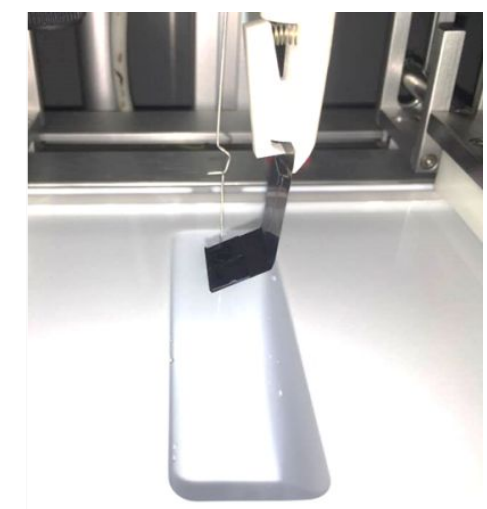

Figure S9. Modified Langmuir-Schaefer technique for the horizontal transfer of monolayers onto different substrates.

\section{Packing considerations according to MMA values}

MMA values are subjected to variations due to various reasons, such as concentration of the stock solution, cleanliness of the water subphase and incorporation of impurities in the monomer solution; due to the high reproducibility of the isotherms obtained in this study, we consider the obtained MMA values to be reliable and useful for a packing discussion of 1 at the air/water interface.

We chose three representative packing models molecule (Figure S10): a) dense packing, b) all-etf packing, c) all-ftf packing. Packing a) is based on a related hydrocarbon molecule and involves $\mathbf{1}$ packing in a dense fashion; ${ }^{4}$ packing b) is based on the model derived from a fragmentary crystal structure solution of 1 discussed previously (Figure S7) in which the anthracene units are all in an edge-to-face relationship; packing $c$ ) is the desired hexagonal packing ought to provide a 2DP, in which 1 packs with all the anthracene units stacking face-to-face. This last model is based on DFT calculations and will be discussed in section 6 . 
a) dense packing

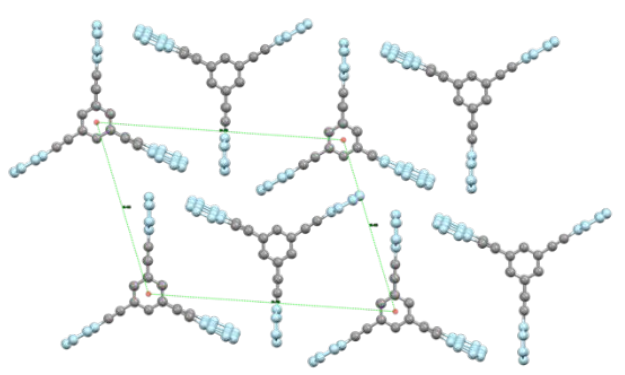

$\mathrm{MMA} \approx 154 \AA^{2}$ b) all-etf packing

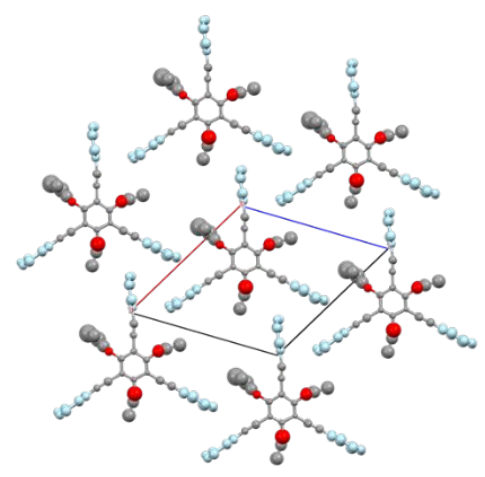

$\mathrm{MMA} \approx 202 \AA^{2}$ b) all-ftf packing

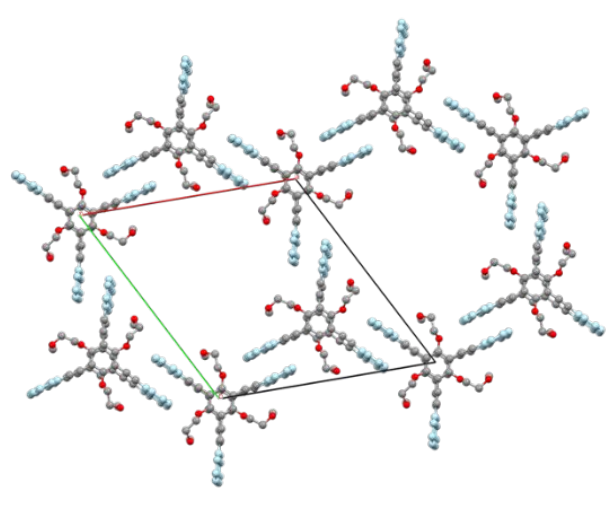

$\mathrm{MMA} \approx 252 \AA^{2}$

Figure S10. Three packing scenarios at the air/water interface and their respective calculated MMA values. Packing a) is based on a crystal structure of a parent hydrocarbon molecule; packing b) is based on a model of a fragmentary crystal structure of 1; packing c) is based on a DFT model. Since in packing a) there are no chains on the central benzene unit, a realistic dense packing for 1 would be expected to have slightly higher MMA values in order to sterically accommodate the substituents.

For packing a) the calculated MMA value is $154 \AA^{2}$ : this value can be considered as the absolute lowest limit for a monolayer of $\mathbf{1}$, since this model is based on a hydrocarbon molecule lacking substituents. In order to sterically accommodate the DEGME chains of $\mathbf{1}$, this packing should expand resulting in higher MMA values. In packing $b$ ) where the anthracene units are in an etf relationship the calculated MMA value is $202 \AA^{2}$ whereas for packing c) it accounts for $252 \AA^{2}$. This last value can be considered as the upper limit for a monolayer of 1 in which the molecules are interacting through their anthracene units. 


\section{Characterization of Langmuir monolayers}

\section{AFM height analysis of the monolayers and film transfer}

Height analysis was performed by using a Nanoscope III Multimode system (Digital Instruments, Santa Barbara, Ca). OMCLAC160TS silicon tips (Olympus, Tokyo, Japan) were used for imaging with a resonance frequency in between 200 and $400 \mathrm{kHz}$ and a spring constant of about $42 \mathrm{~N} / \mathrm{m}$. The Langmuir films were transferred horizontally on 285 $\mathrm{nm} \mathrm{SiO}{ }_{2}$-coated silicon wafers. Height analysis was performed either at cracked regions of the films or at artificially created step edges (by covering part of the wafers with a piece of freshly cleaved mica before transferring the monolayer).

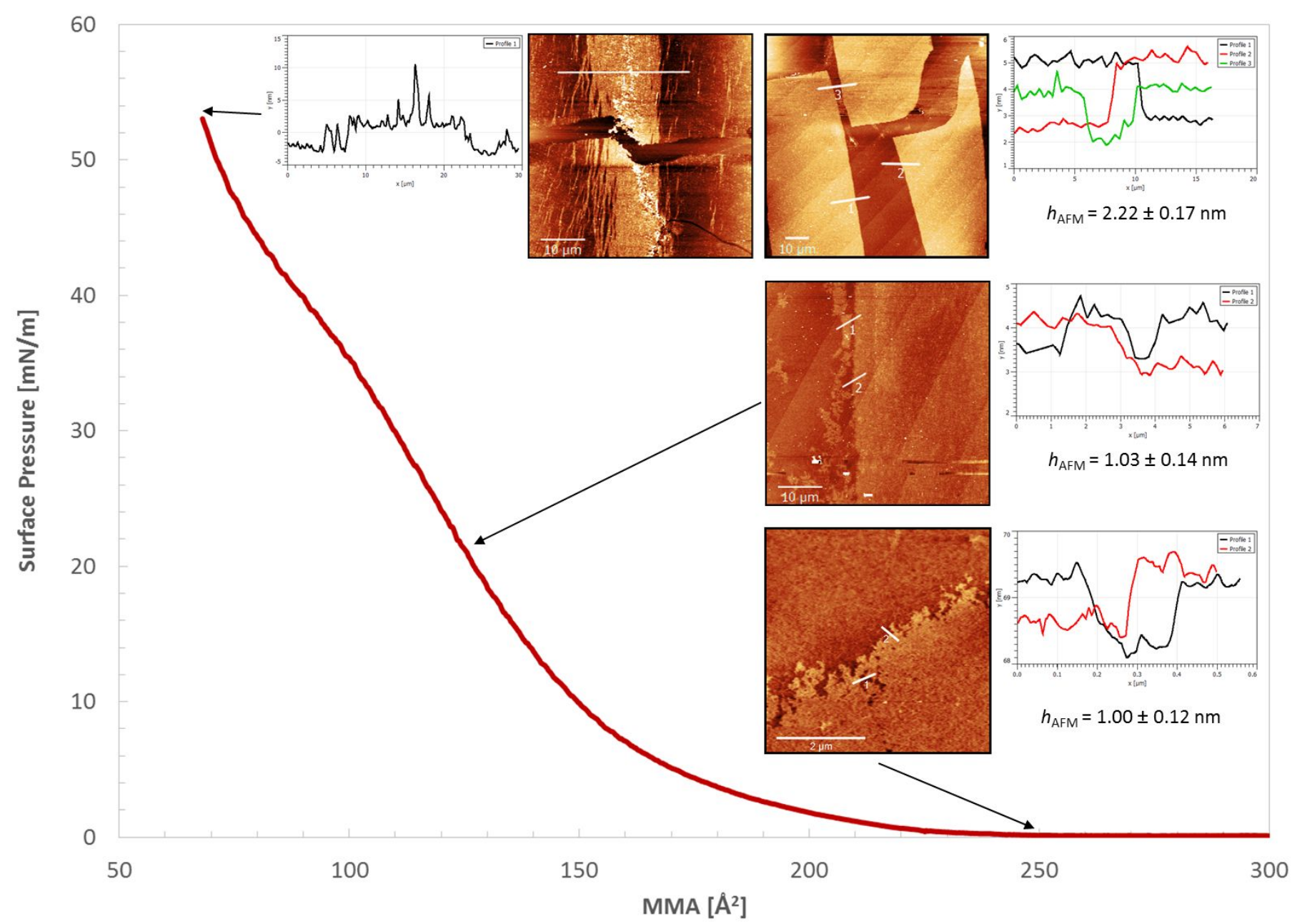

Figure S11. Tapping mode AFM height images and height profiles of the monomer film transferred onto silicon substrates at different surface pressures. At SP of $0.2 \mathrm{mN} / \mathrm{m}$ and $20 \mathrm{mN} / \mathrm{m}$ the film thickness is approximately $1 \mathrm{~nm}$, corresponding to monolayers. Above $30 \mathrm{mN} / \mathrm{m}$ the monolayers start to collapse as indicated from the flexion on the isotherm resulting in double- and multilayers at SP of $52 \mathrm{mN} / \mathrm{m}$. Corrugation is some regions of the fully compressed film is clearly visibly, reaching thicknesses of $17 \mathrm{~nm}$. In the uncompressed form, monolayers of 1 seem to selfassemble in islands with structural integrity. 


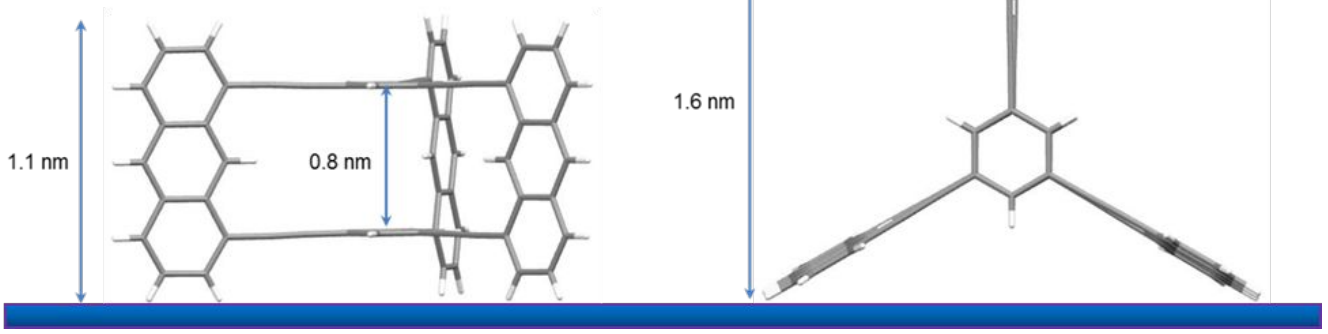

Figure S12. Molecular model of 1 (DEGME chains omitted for clarity) with calculated dimensions for different orientations on a substrate. The measured thickness $h_{\mathrm{AFM}}$ of the monolayers is approximately $1.0 \mathrm{~nm}$, which is in good agreement with the calculated height $h_{\text {calc }}$ for the monomer standing with the anthracenes perpendicular to the substrate. Other orientations would give higher thickness values.

\section{In-situ Brewster's angle microscopy}

To visualize the films by Brewster angle microscopy (BAM), a KSV MicroBAM operating by a $659 \mathrm{~nm}$ laser was used.
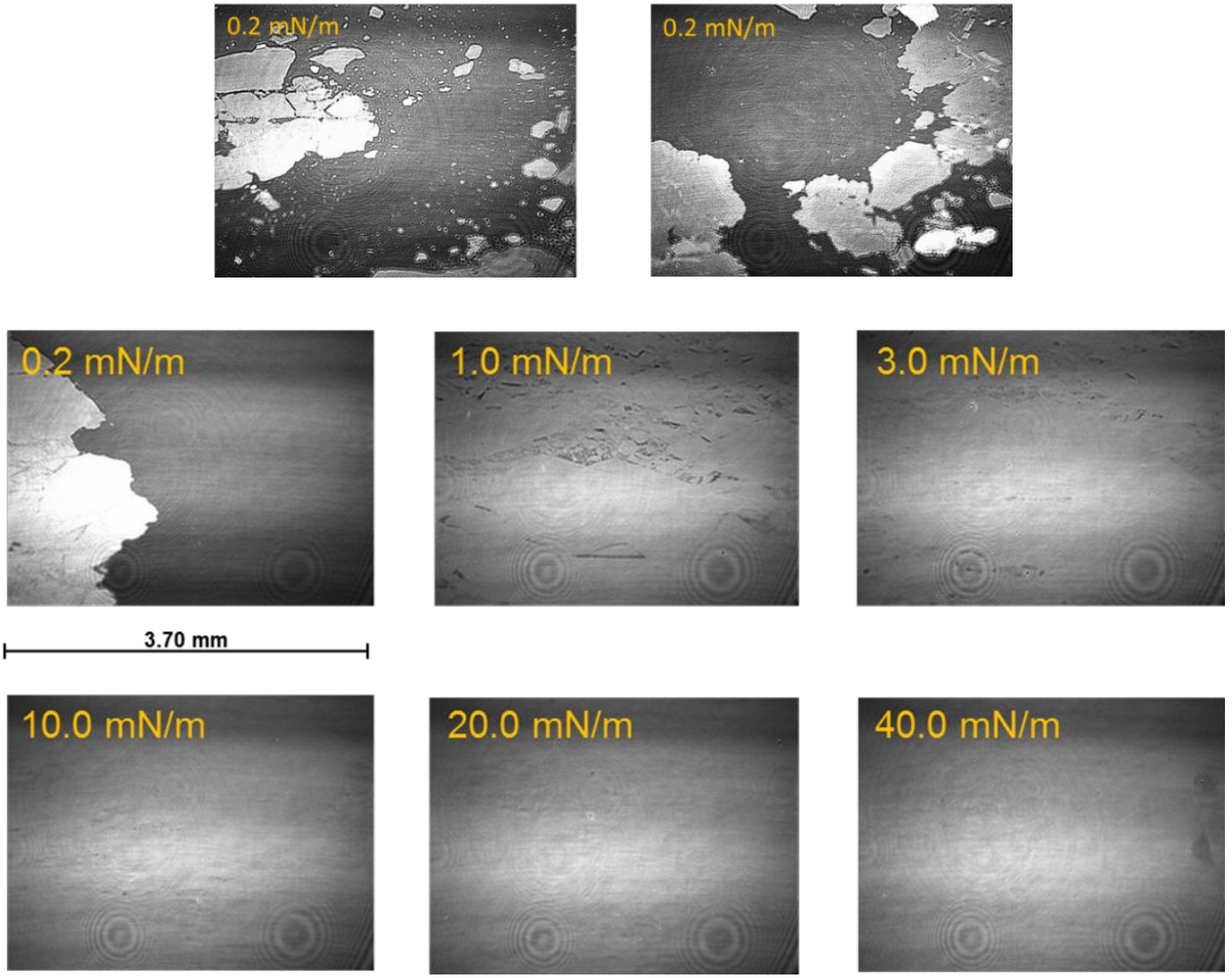

Figure S13. BAM micrographs recorded in the uncompressed state $(0.2 \mathrm{mN} / \mathrm{m})$ and at different surface pressures during the compression process. In the uncompressed state, islands are clearly visible, which upon compression coalesce into a lateral homogeneous film with homogeneous thickness. Recording an image at surface pressures above $40 \mathrm{mN} / \mathrm{m}$ is not possible due to the geometry of the optomechanical components of the microscope. Note: Due to automatic adjustment of the contrast in each image, brightness level is only meant to be studied in images individually and not to be compared with other recorded images. 


\section{Visualization of the monolayers at the interface}

To visualize the monolayers by naked eye, a standard portable laboratory UV lamp for TLC (Camag) was used at 254 $\mathrm{nm}$.
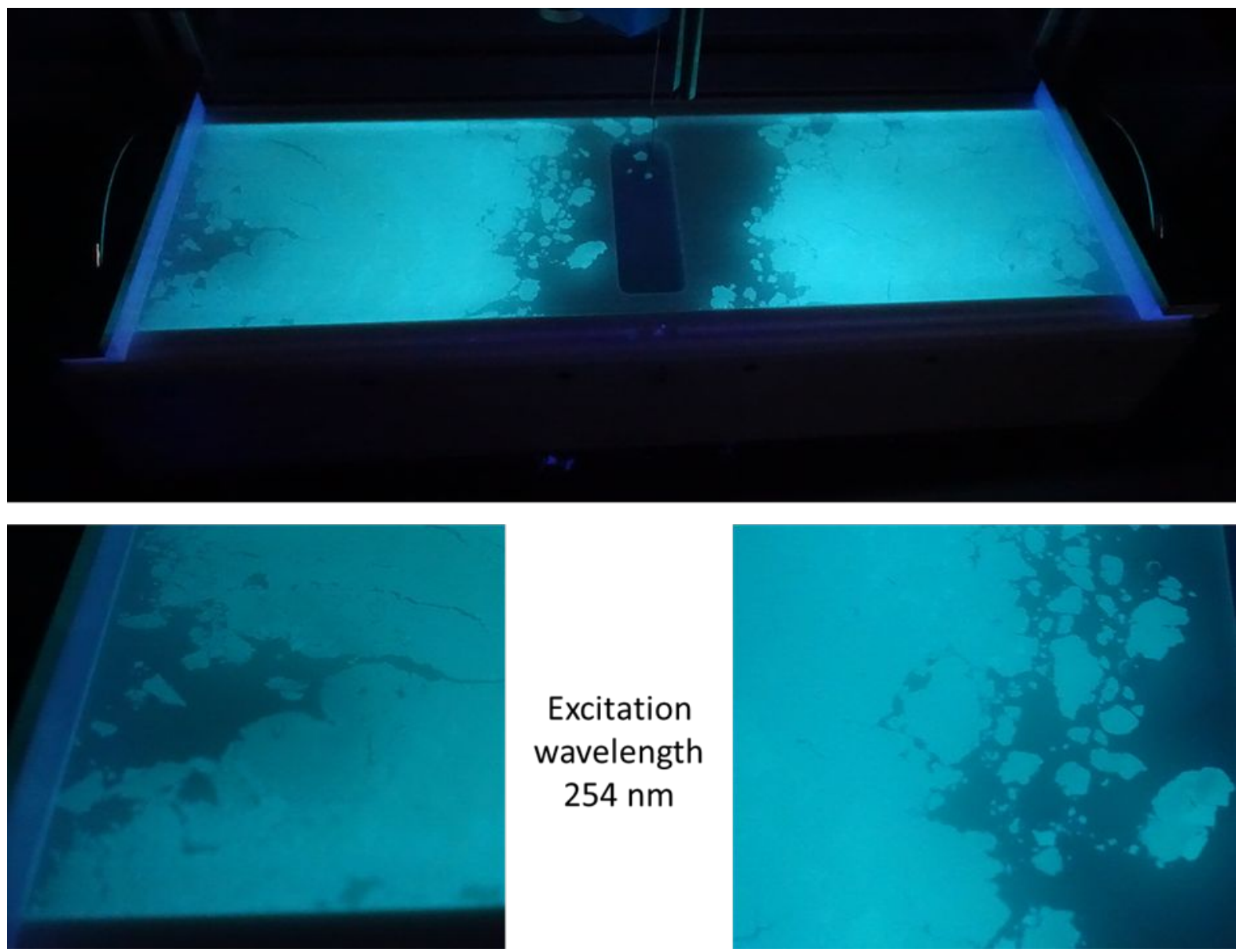

Figure S14. Compound 1 after being spread at the air/water interface: the monolayer can be visualized by naked eye upon excitation at $254 \mathrm{~nm}$ with a standard UV lamp. 


\section{UV/Vis absorption and emission spectra in solution and on quartz}

UV/Vis absorption spectra were recorded with a JASCO V-670 UV-Vis-NIR spectrophotometer using a quartz cell with a path length of $1 \mathrm{~cm}$. Emission spectra were recorded with a Spex Fluorolog 2 spectrophotometer from Jobin Yvon (United Kingdom) using a quartz cell with a path length of $1 \mathrm{~cm}$ by diluting by a factor of 30-60 (depending on the compound) the solutions employed for the UV/Vis absorption measurements.
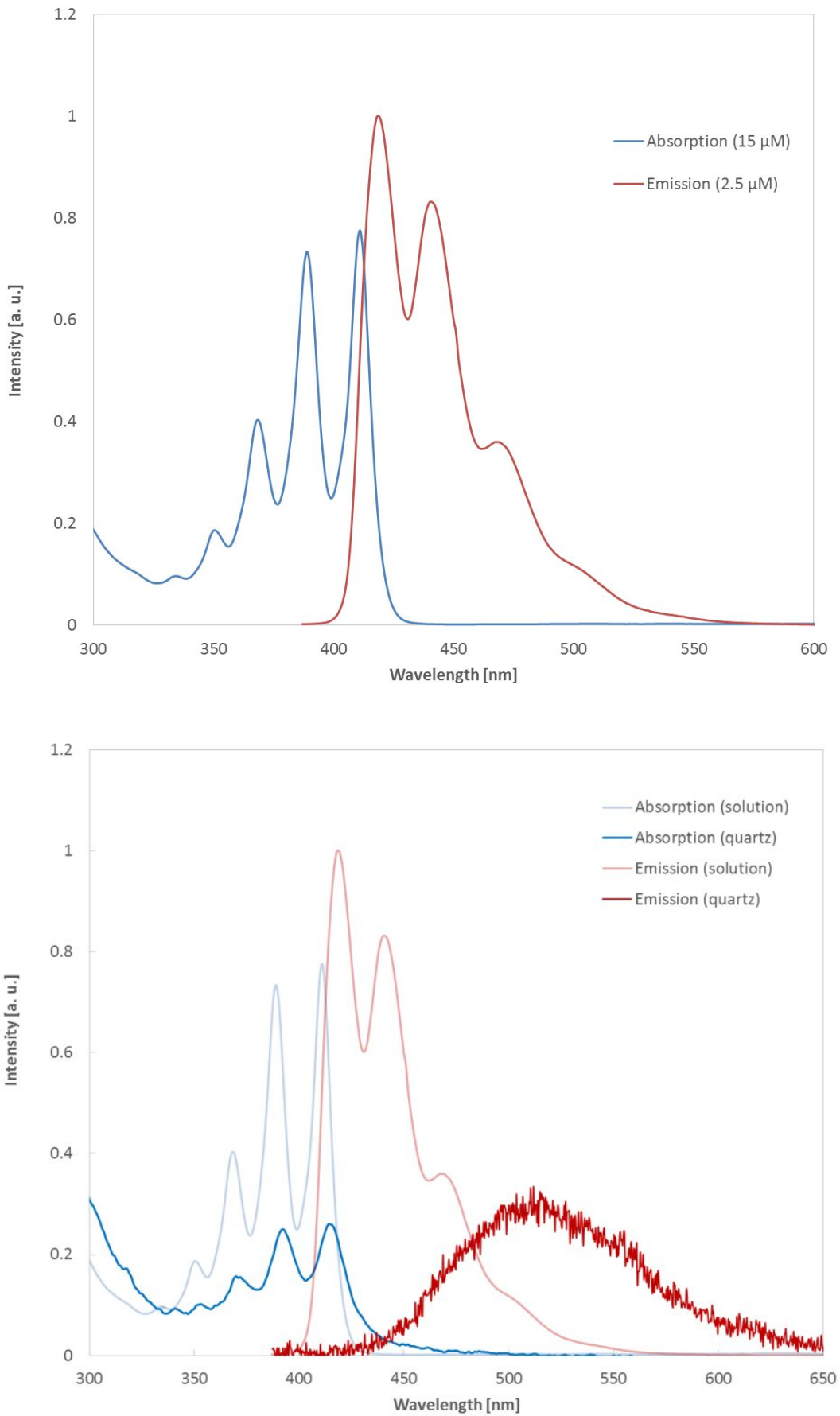

Figure S15. UV/Vis absorption and emission spectra of a monomer solution in chloroform (top) and of an uncompressed monolayer transferred on quartz (bottom). On quartz the absorption vibronic bands are slightly redshifted by $3 \mathrm{~nm}$ whereas in the emission spectrum the vibronic signature is completely lost resulting in a pure excimer emission centered at around $510 \mathrm{~nm}$. 


\section{In-situ fluorescence spectroscopy at the interface}

For recording the spectra from the interface, an Acton series spectrometer from Princeton Instruments (NJ, USA) was used. The spectrometer was equipped with an SP-2556 Acton Research 500 mm Imaging Spectrograph (Acton, MA, USA), FC fiber stage (modified in house to be $3 \mathrm{~mm}$ closer to the reflecting mirror) and three standard gratings with groove densities of $1200 \mathrm{~mm}^{-1}, 600 \mathrm{~mm}^{-1}$ and $150 \mathrm{~mm}^{-1}$, with blaze size of $500 \mathrm{~nm}$ and $68 \mathrm{~mm} \times 68 \mathrm{~mm}$ dimensional size. The CCD camera mounted on the spectrograph was a Princeton Instruments PIXIS 256E (NJ, USA). The read-out fiber was purchased from AFW Technologies Pty Ltd (Hallam, Australia) and had a core diameter of 50 $\mu \mathrm{m}$, numerical aperture of 0.12 and FC connectors on both ends. The light source used for excitation of the monomers on the interface was an LED with $\lambda=365 \mathrm{~nm}$ and with $250 \mathrm{~mW}$ power, which was purchased from Omicron Laser (Rodgau, Germany). It was mounted on a lens tube along with a UV fused silica bi-convex lens with $40 \mathrm{~mm}$ focal length (Thorlabs, LB4030-UV, Newton, NJ, USA) as well as a band-pass filter transmitting at $370 \mathrm{~nm}$ with FWHM of $10 \mathrm{~nm}$ (Thorlabs, FB370-10, Newton, NJ, USA). The beam was focused the interface to produce an illuminating spot with a diameter of about $2 \mathrm{~mm}$. The read-out stage was aligned and focused so to collect as much emission signal as possible. The read-out fiber was fixed on a stage which was built in-house. The exposure time for simple measurements was 30 seconds whereas for the fluorescence decay experiment it was 60 seconds for each spectrum while the LED was at full power during the acquisition time. The delay time between the spectra was 2 minutes. A binning of 4 was used to record the data. The CCD read-out was set to the region of interest (ROI), while the ROI set-up on the slit ran from 130 as the start point with a height of 23. During the course of the fluorescence decay experiment the entire set-up (LED for excitation and detector for collecting the fluorescence emission) was kept in a fixed geometry. The set-up for the experiment is shown in Figure S16.

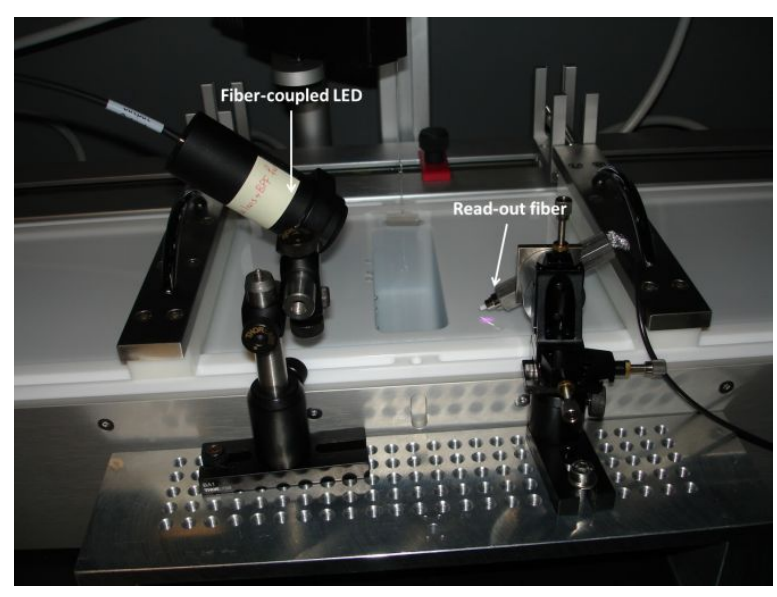

Figure S16. Experimental set-up for in-situ fluorescence measurements at the air/water interface. 


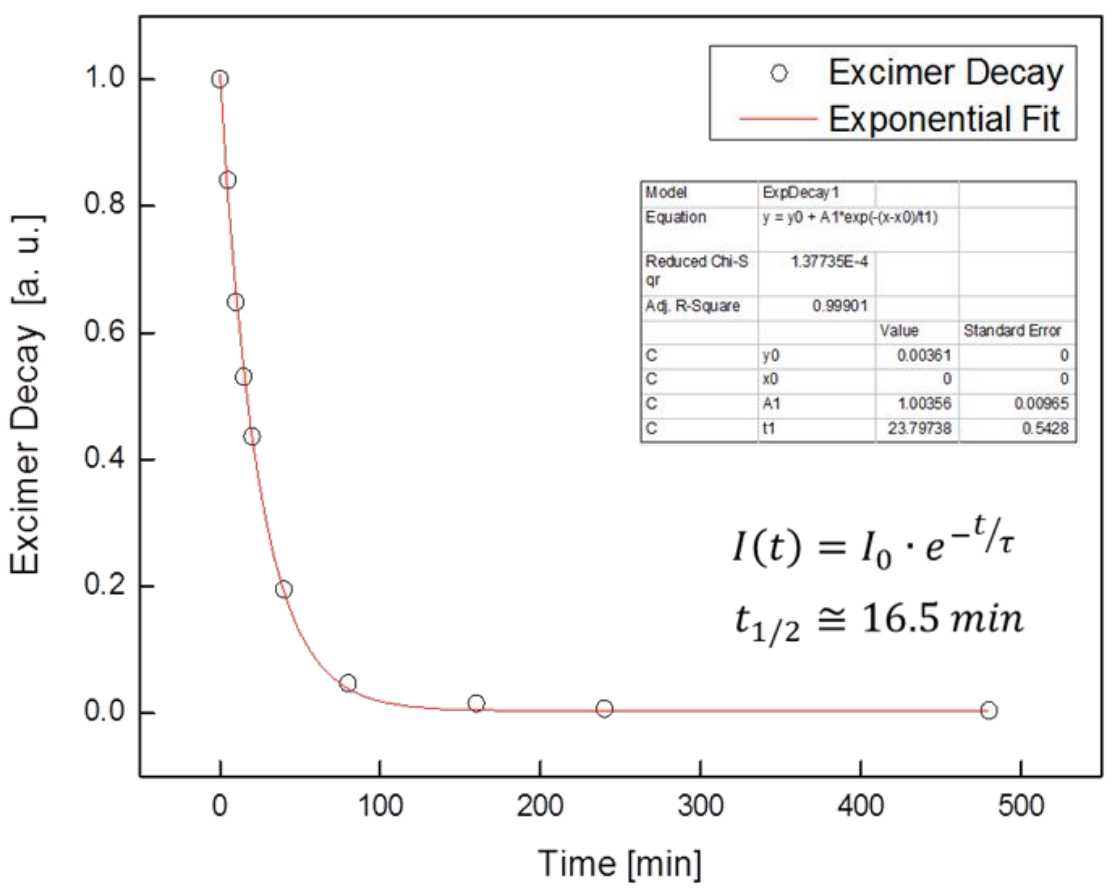

Figure S17. Exponential decay of the excimer fluorescence $\left(\lambda_{\text {MAX }}\right)$ upon irradiation at $365 \mathrm{~nm}$ with a $250 \mathrm{~mW}$ fibercoupled LED. The decay follows first order kinetics and has a half-life of $16.5 \mathrm{~min}$.

\section{Polymerization at the air/water interface}

The monomer monolayers were polymerized by direct exposure to a custom-made $385 \mathrm{~nm}$ LED with $1 \mathrm{~W}$ of nominal power (Figure S18). The irradiation areas could be varied by varying the distance of the LED from the interface.

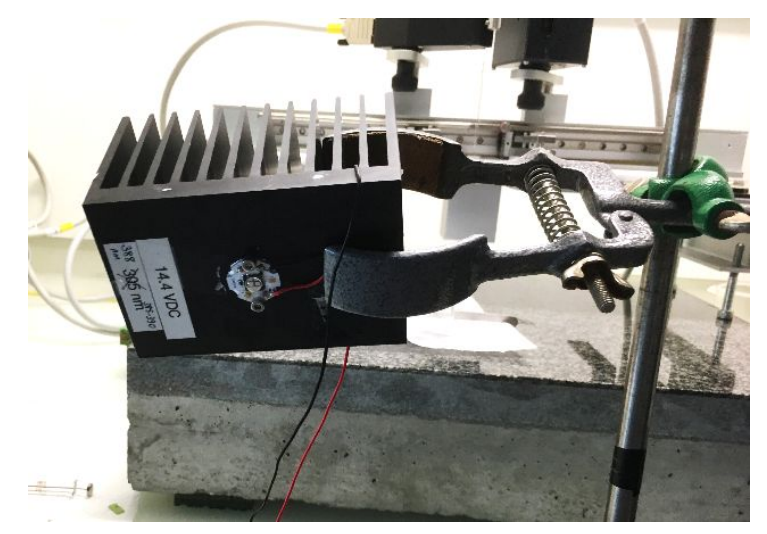

Figure S18. Custom-made $385 \mathrm{~nm} 1$ W LED used for the polymerizations at the air/water interface. 


\section{Tip-Enhanced Raman Spectroscopy (TERS)}

The template stripped Au substrate is prepared based on the same procedure as published in this literature. ${ }^{8}$ The freshly stripped Au substrate was used to transfer the monolayer sheet (as described at page S17) from the air-water interface to the air followed by TERS measurements. STM-TERS measurements was performed on a top illumination TERS instrument that combines STM with a Raman spectrometer (NT-MDT, Russia, NTEGRA Spectra Upright). An electrochemically etched Ag tip was used to obtain both the topography and TER spectra. The $632.8 \mathrm{~nm}$ He-Ne laser was used as an excitation source. For all the measurements, the tunneling conditions were 200 pA and $0.2 \mathrm{~V}$, the intensity of laser was $30 \mu \mathrm{W}$ with 1s exposure time. TERS peak assignment was performed using literature values for related anthracene monomers. ${ }^{9}$

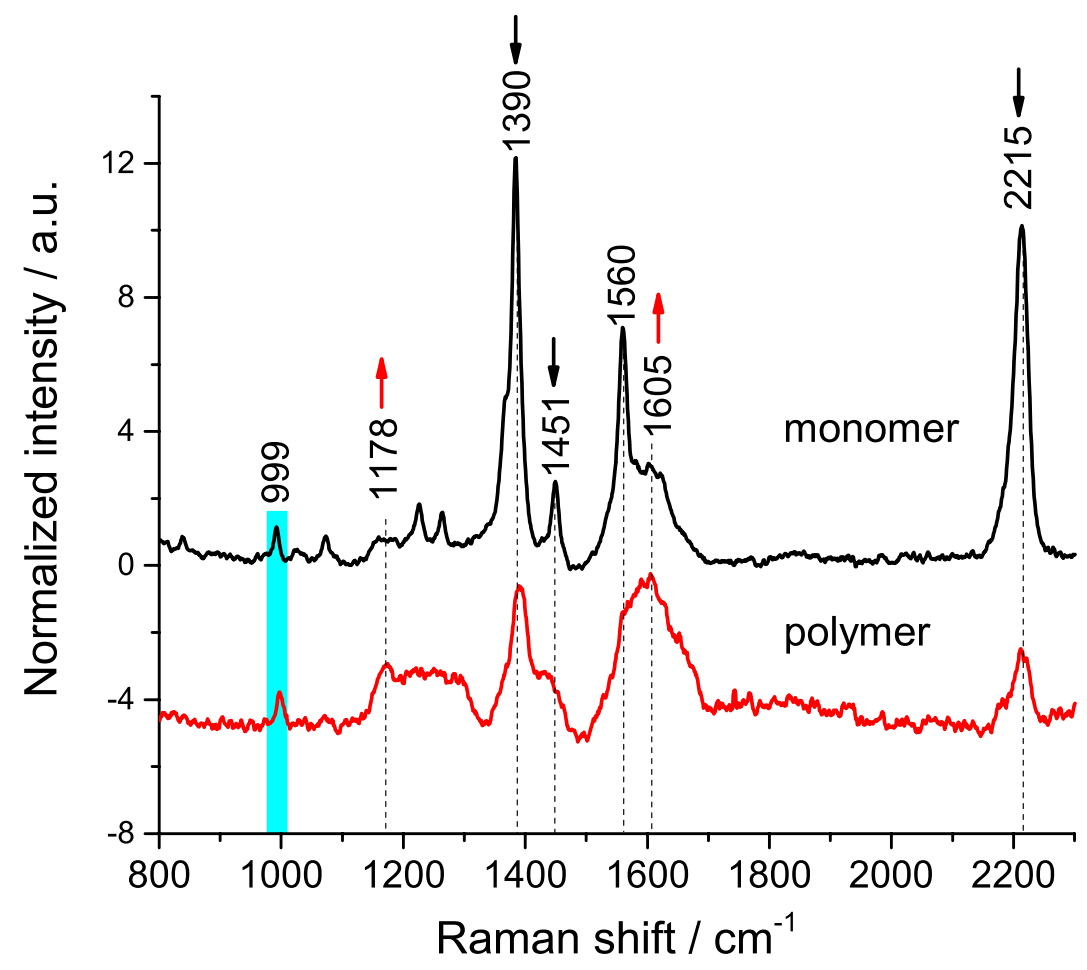

Figure S19 Baseline corrected average TER spectra (normalized by the respective Raman intensity of the peak at 999 $\mathrm{cm}^{-1}$ ) of monomer and polymer monolayers from corresponding TERS maps with $10 \times 10$ pixels (100 spectra). The baseline corrections were performed by Origin 9.3 software by the 2nd derivative method with 15 anchor points. The intensive Raman peak at $1390 \mathrm{~cm}^{-1}$ of monomer corresponds to the characteristic $\mathrm{v}(\mathrm{C}=\mathrm{C})$ mode of anthracene. After irradiation of $2 \mathrm{~h}$, the intensity of this peak is significantly decreased. The Raman peak at $999 \mathrm{~cm}^{-1}$, which presents on both spectra of monomer and polymer, is the breathing mode of benzene ring in the middle of the monomer. This peak is basically unaffected after polymerization and was therefore selected as internal reference. Based on the intensity decrease of anthracene peak referring to the reference peak, we can estimate the conversion degree of monomer into polymer after UV irradiation for $2 \mathrm{~h}$. The peak intensity ratios between anthracene peak and reference peak are listed in the Table S1. The calculation detail of polymerization is shown below:

Polymerization degree $=1-4.28 / 24.3=82.4 \%$ 
Table S1. Peak intensity ratios between anthracene peak and ring breathing mode of benzene ring of monomer and polymer

\begin{tabular}{c|c|c}
\hline & \multicolumn{2}{|c}{ Peak intensity ratio } \\
\hline Sample & $\begin{array}{c}1390 \mathrm{~cm}^{-1} / \\
995 \mathrm{~cm}^{-1}\end{array}$ & $\begin{array}{c}\text { Polymerization } \\
\text { degree / \% }\end{array}$ \\
\hline Monomer & 24.3 & \\
\hline $\begin{array}{c}\text { Polymer with irradiation time } \\
\text { of 2 } \mathrm{h}\end{array}$ & 4.28 & 82.4 \\
\hline
\end{tabular}

\section{Scanning electron microscopy (SEM)}

After polymerization, TEM grids made of copper with the mesh size of 1000 (PLANO, G2780C) were placed on the irradiated spot on the surface of the water. A clean white piece of paper was then gently put on the grids so for them to adsorb on it. The paper along with the grids on it was then removed by hand and dried overnight at room temperature. The TEM grids were placed on a PLANO G3662 holder and imaged with FEG-SEM, Zeiss LEO Gemini 1530, Germany, microscope with an in-lens detector.

\section{X-ray Photoelectron Spectroscopy (XPS)}

The samples were characterized by X-ray photoelectron spectroscopy (XPS) using a Quantum 2000 (Physical Electronics Inc.) instrument under ultrahigh vacuum (<5 $\times 10-7 \mathrm{~Pa})$. Monochromatic Al Ka X-rays with a photon energy hv $=1486.7 \mathrm{eV}$ were used, and the data were recorded at an analyzer pass energy of $23.50 \mathrm{eV}$ and a step size of $0.1 \mathrm{eV}$ (for the detailed spectra) and a pass energy of $58.70 \mathrm{eV}$ and a step size of $0.5 \mathrm{eV}$ for the survey. Argon ions and electron neutralizers were used to compensate for surface charging. The binding energy of Au $4 f$ peaks of the Au layer, onto which the polymer films were deposited, correspond to literature values. CasaXPS software was used for data processing. After subtracting a Shirley background, the integrated intensities of the O1s and C1s peaks yield the ratio of the total oxygen to carbon concentration. The main outcome is that the $\mathrm{O} 1 \mathrm{~s} / \mathrm{C} 1 \mathrm{~s}$ ratio remains constant with the uncertainty of the measurement for illumination times up to $120 \mathrm{~min}$. The increase at long exposure time may be indication of a degradation of the film. 

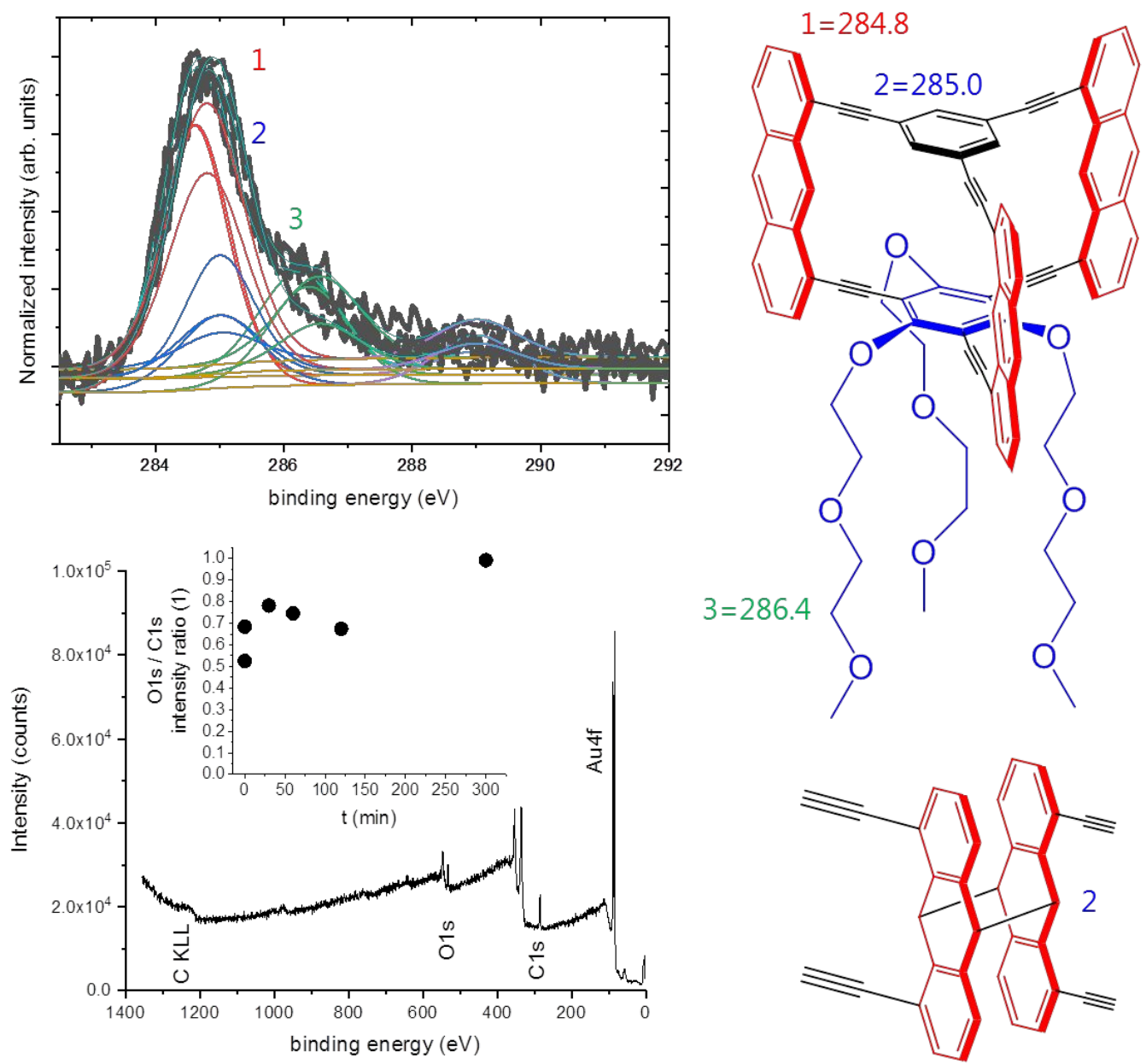

Figure S20. XPS analysis of the polymer monolayers at the different irradiation times. The carbon 1s peak can be deconvoluted into at least contributions from aromatic carbons (1) and cyclic carbons bridging the molecules after polymerization (2). The triple bonds overlap with the bridging carbons. Unfortunately, the chemical shifts of these atoms are very small and impeded a reliable distinction from data fits. The carbons in the DEGME chains are clearly separated (3). 


\section{Optically-Rewritable Molecular Paper}

Writing at the air/water interface with a $405 \mathrm{~nm}$ laser pointer

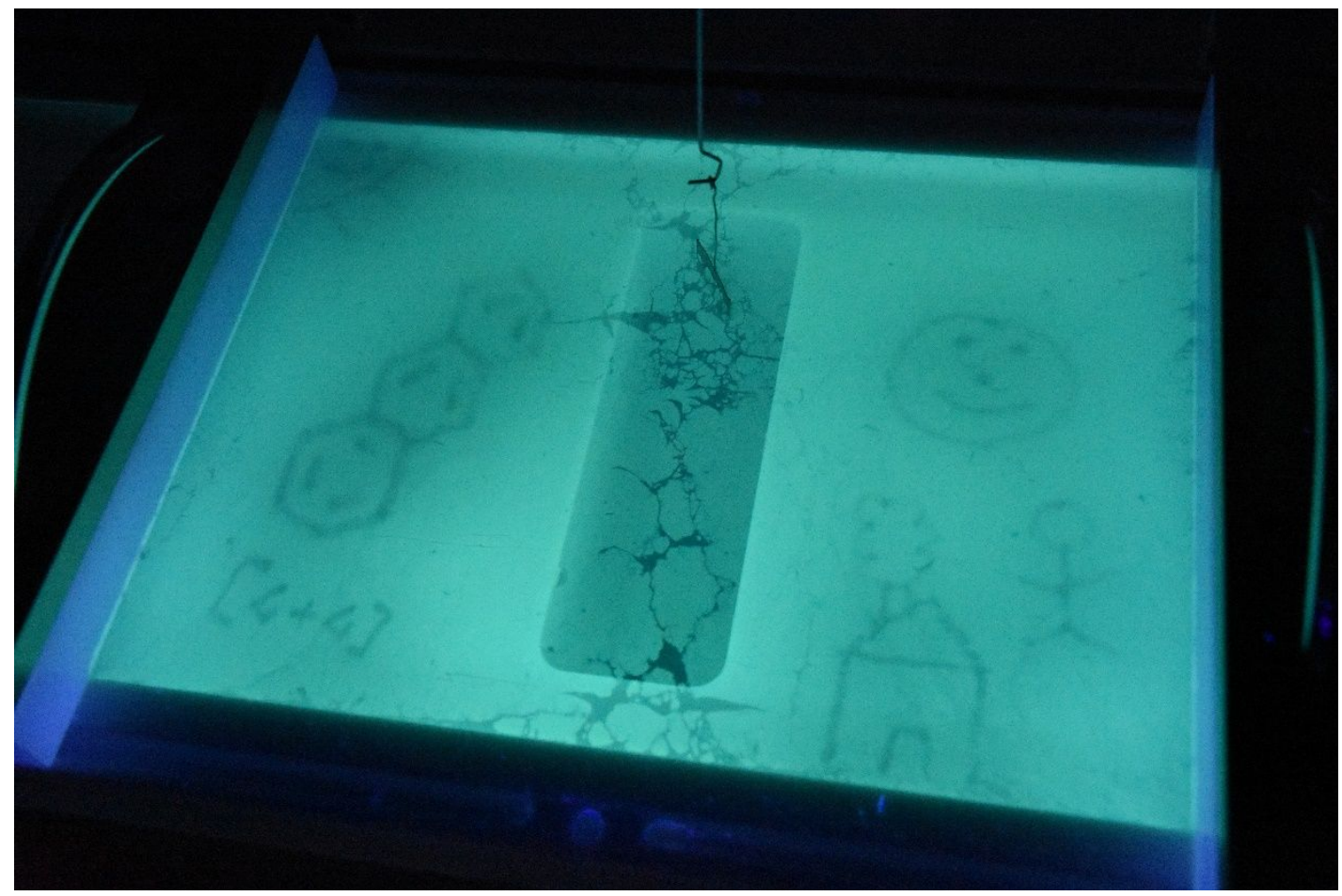

Figure S21. Writing on a monomer monolayer with a $405 \mathrm{~nm}$ laser (200 mW) pointer.

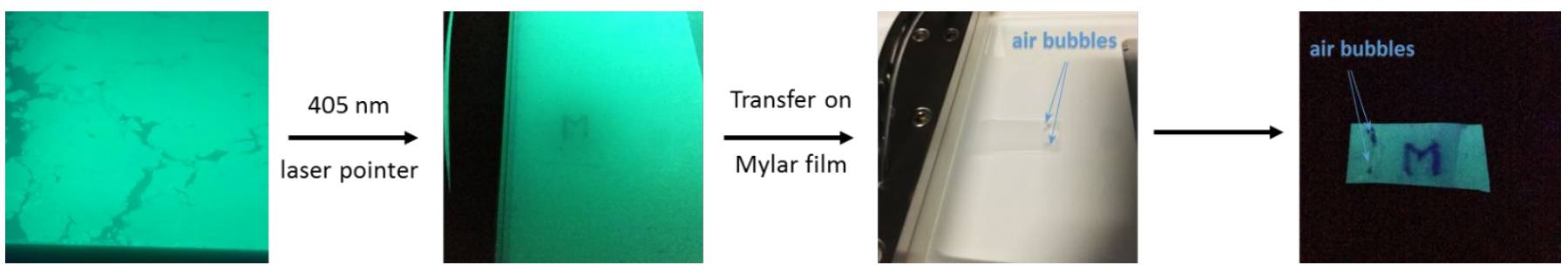

Figure S22. Writing on a monomer monolayer at the air/water interface with a $405 \mathrm{~nm}$ laser (200 $\mathrm{mW})$ pointer and subsequent transfer of the pattern " $\mathrm{M}$ " on Mylar film. The writing occurs in seconds and the monolayer immediately and efficiently sticks on Mylar. 


\section{Confocal Laser Scanning Microscopy (CLSM)}

A Zeiss LSM 780 laser scanning confocal microscope (Oberkochen, Germany) was used. The system is equipped with a motorized $X-Y$ stage $(135 \mathrm{~cm} \times 80 \mathrm{~cm})$ and two conventional PMTs and a highly sensitive, multiarray 32PMT GaAsP detector for fast spectral scanning. Images were recorded using a laser line of $405 \mathrm{~nm}$ (diode) and a 10X, 0.3NA EC Plan-Neofluar Ph1 M27 objective. Lambda scans were conducted using a laser line of 405 nm, a filter of 446-695 nm and a lambda window distance of $8.9 \mathrm{~nm}$. Data processing was carried out using the ZEN Black 2012 software by Zeiss (Oberkochen, Germany) and Fiji (U.S. National Institutes of Health, Bethesda, Maryland, USA). Contrast and/or Brightness of images were increased to obtain optimal image quality. The monolayers were transferred on $285 \mathrm{~nm}$ $\mathrm{SiO}_{2}$-coated silicon wafers.
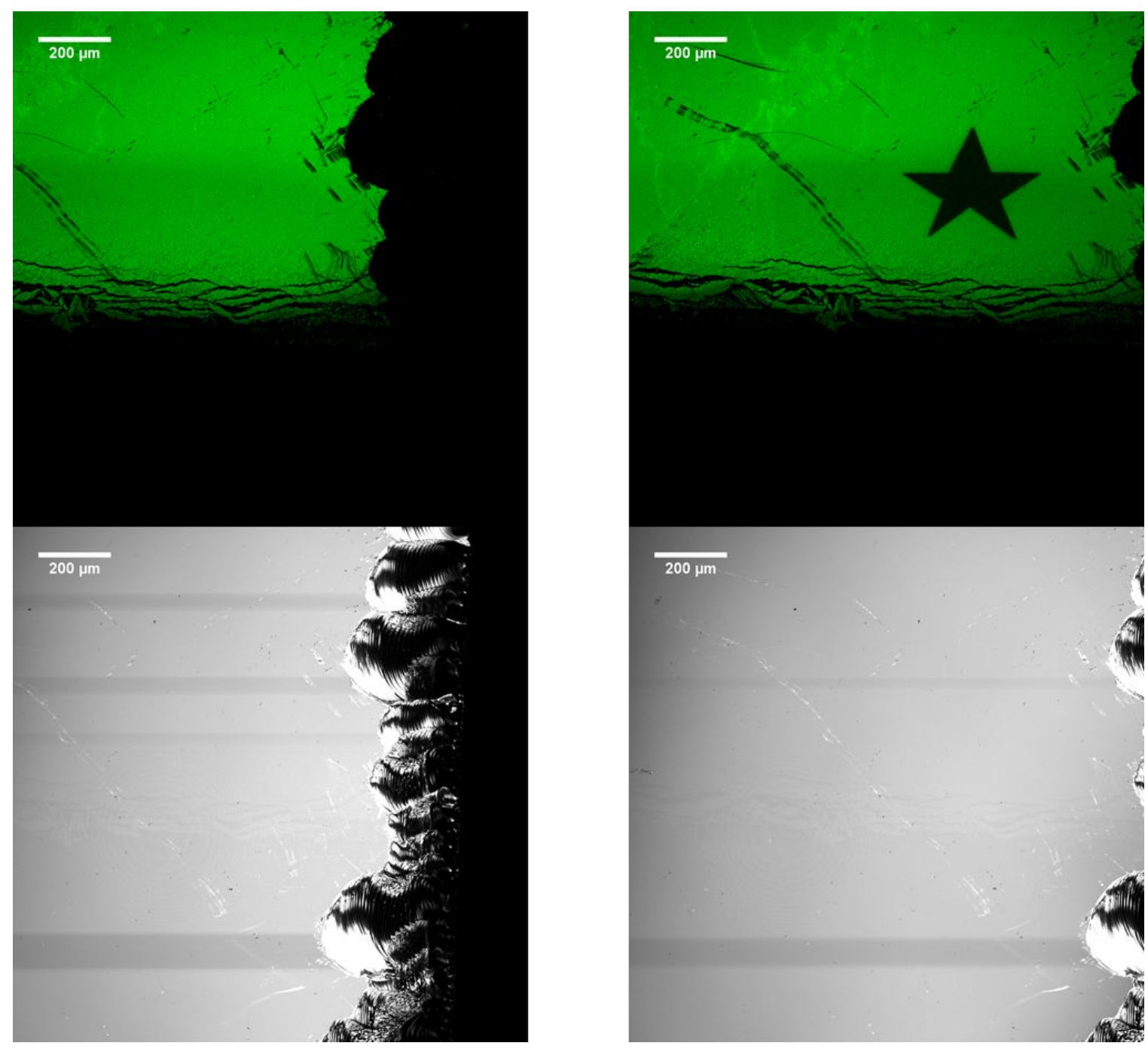

Figure S23. Optical micrographs of the monomer monolayer transferred on a $285 \mathrm{~nm} \mathrm{SiO}{ }_{2}$-coated silicon wafer visualized by CLSM before (left) and after bleaching a star (right). The pictures are obtained by scanning with a 405 $\mathrm{nm}$ laser (top images) and $633 \mathrm{~nm}$ laser (bottom images). The bleaching was done with a $405 \mathrm{~nm}$ laser. 


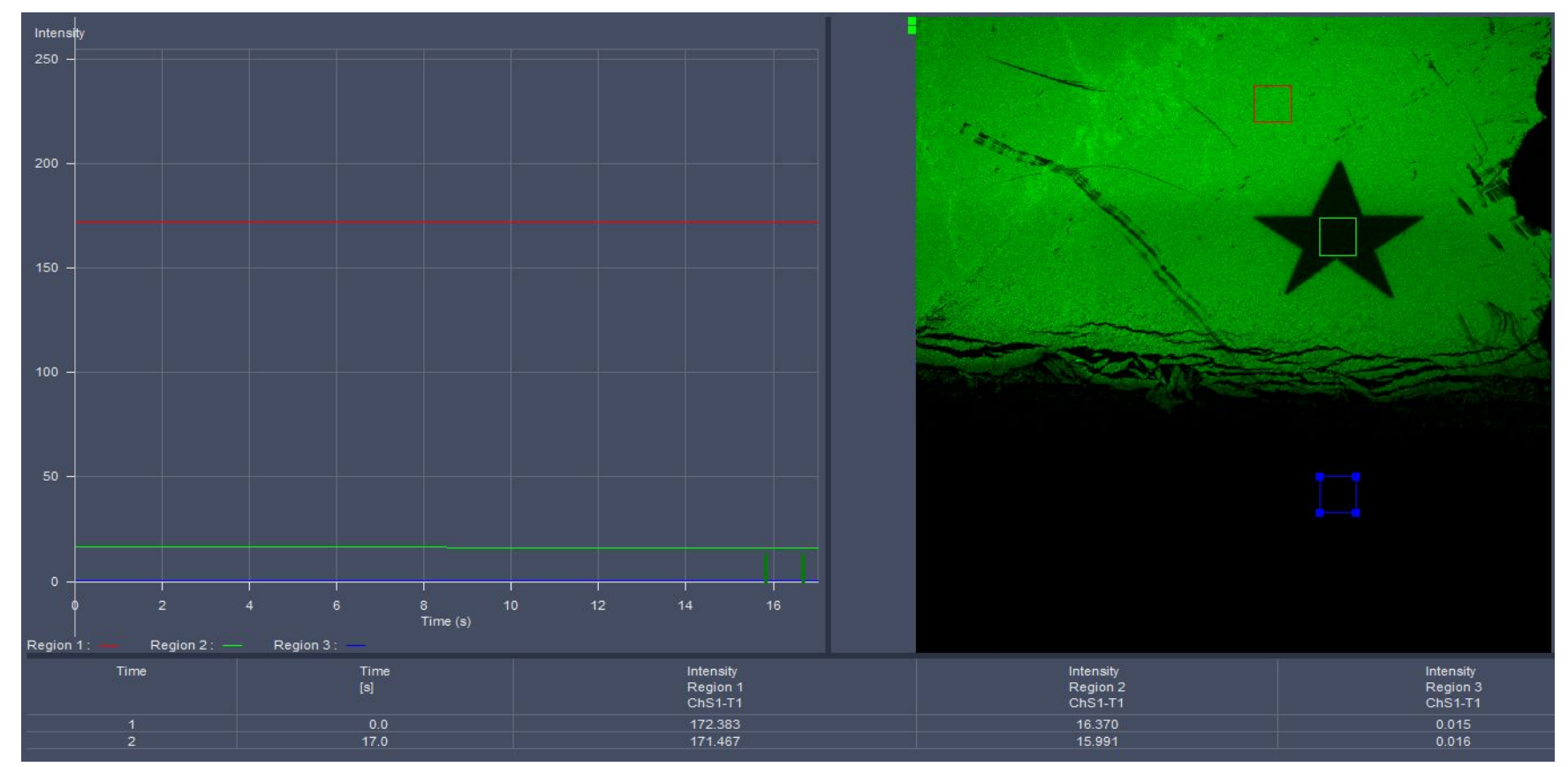

Figure S24. Fluorescence intensities in non-irradiated monolayer (red), bleached region (green) and control region without any monolayer (blue). The bleaching process decreases the fluorescence by $90 \%$.

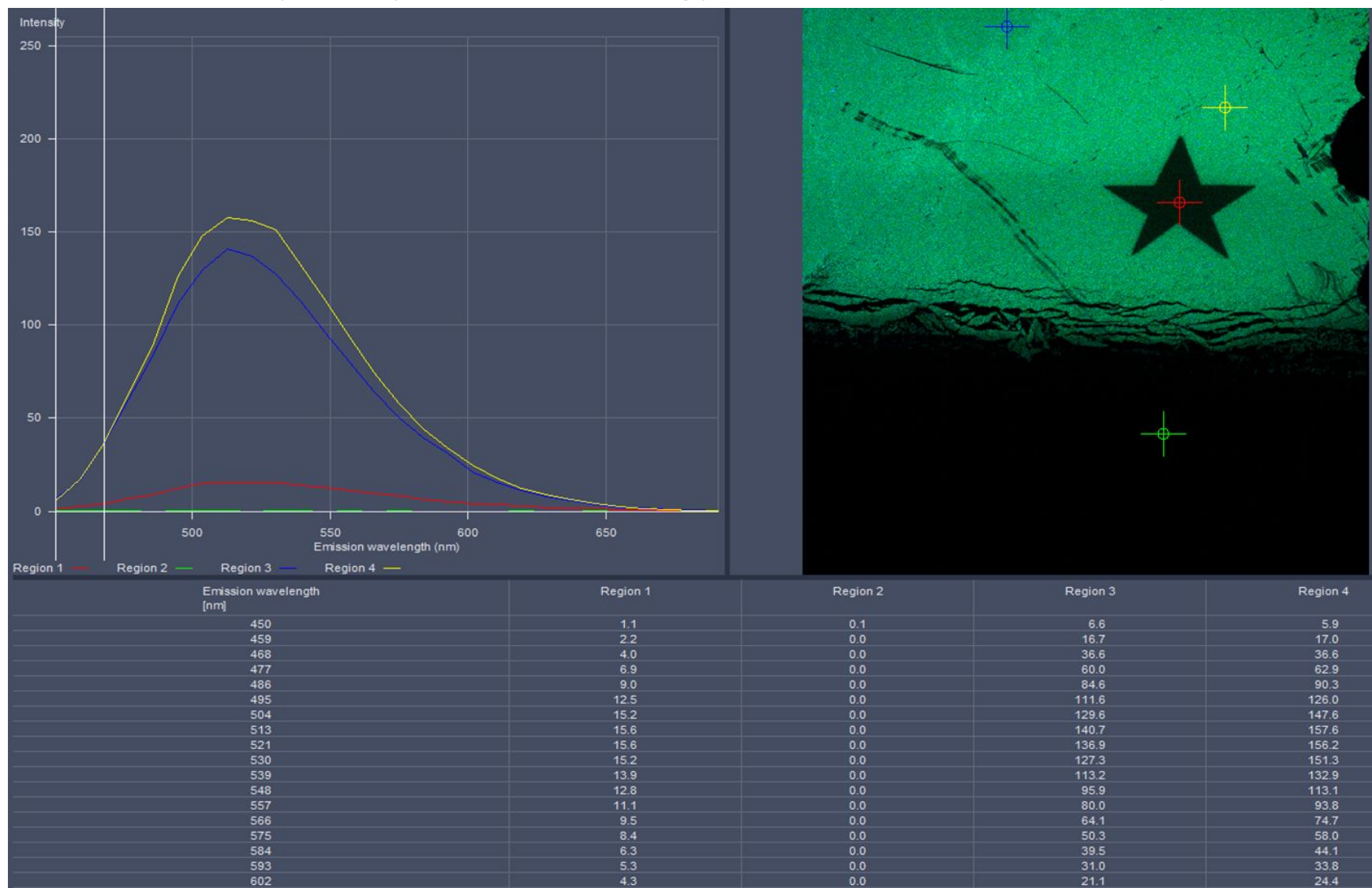

Figure S25. Lambda scans at different regions of the monolayer: non-irradiated regions (blue and yellow), bleached region (red) and control region (green). The bleaching process decreases the emission by $90 \%$. The emission of the monolayer deposited on silicon oxide wafers corresponds to an excimer emission centered at around $513 \mathrm{~nm}$, very similar to the emission observed at the air/water interface: from this one can conclude that during the transfer process on the wafer, the packing remains basically unaffected. 

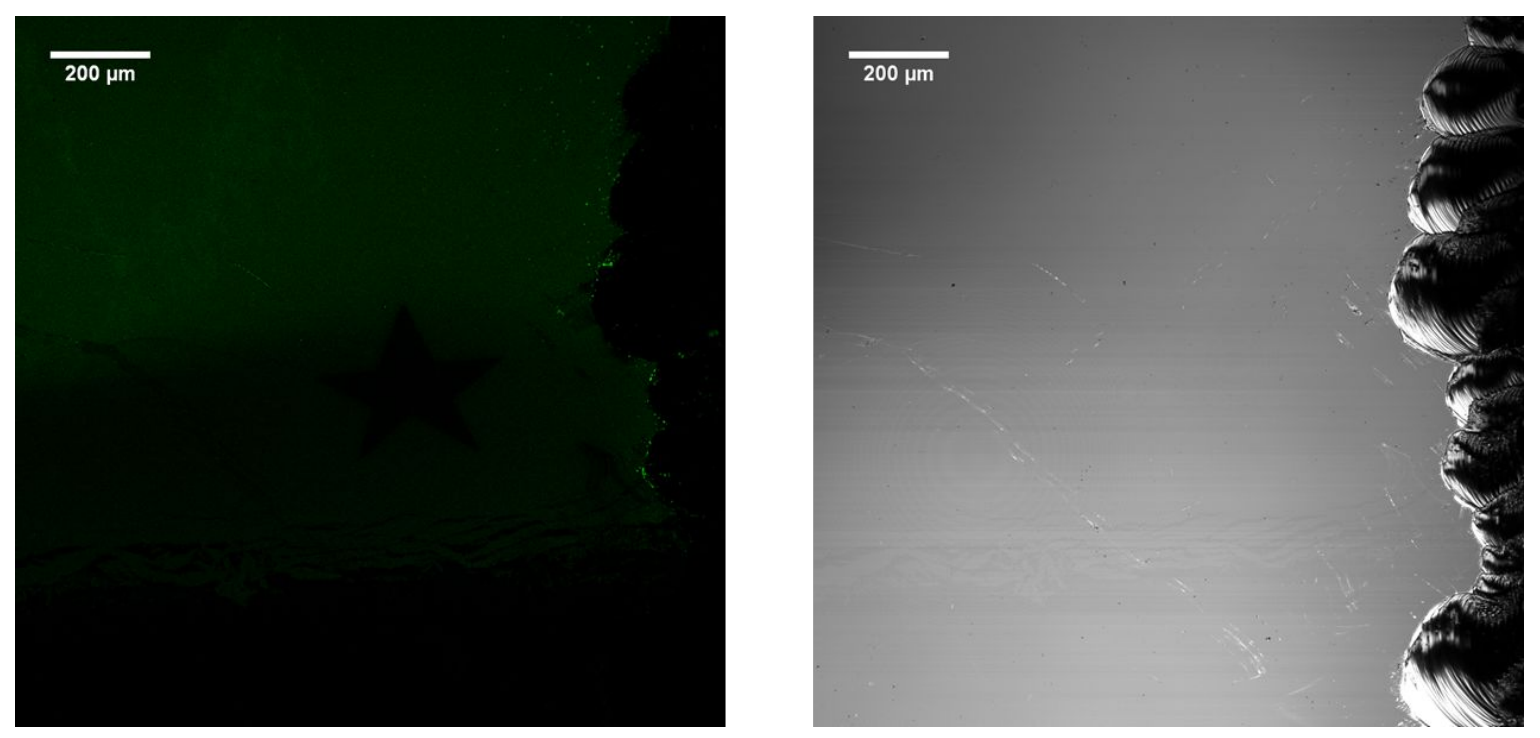

Figure S26. Monolayer after treatment at $200^{\circ} \mathrm{C}$ under nitrogen for 4 hours with the same detector settings used for a fresh monolayer. The fluorescence intensity is drastically decreased probably due to thermal degradation.
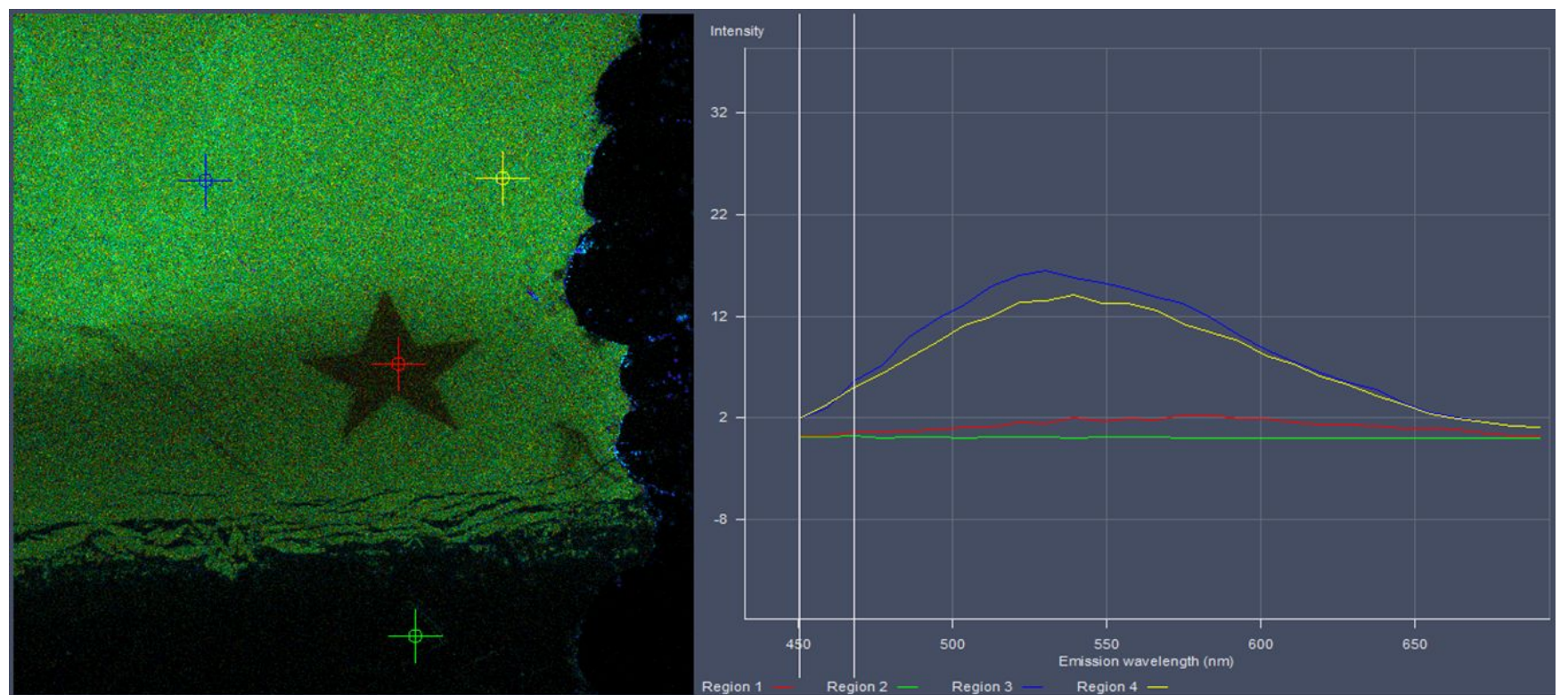

Figure S27. Same monolayer as before but visualized with increased detector's sensitivity. The overall fluorescence is decreased by approximately $90 \%$ due to thermal degradation (compared with the values in Figure S25). The patterned star is still present. Non-irradiated regions (blue and yellow), bleached region (red) and control region (green). 


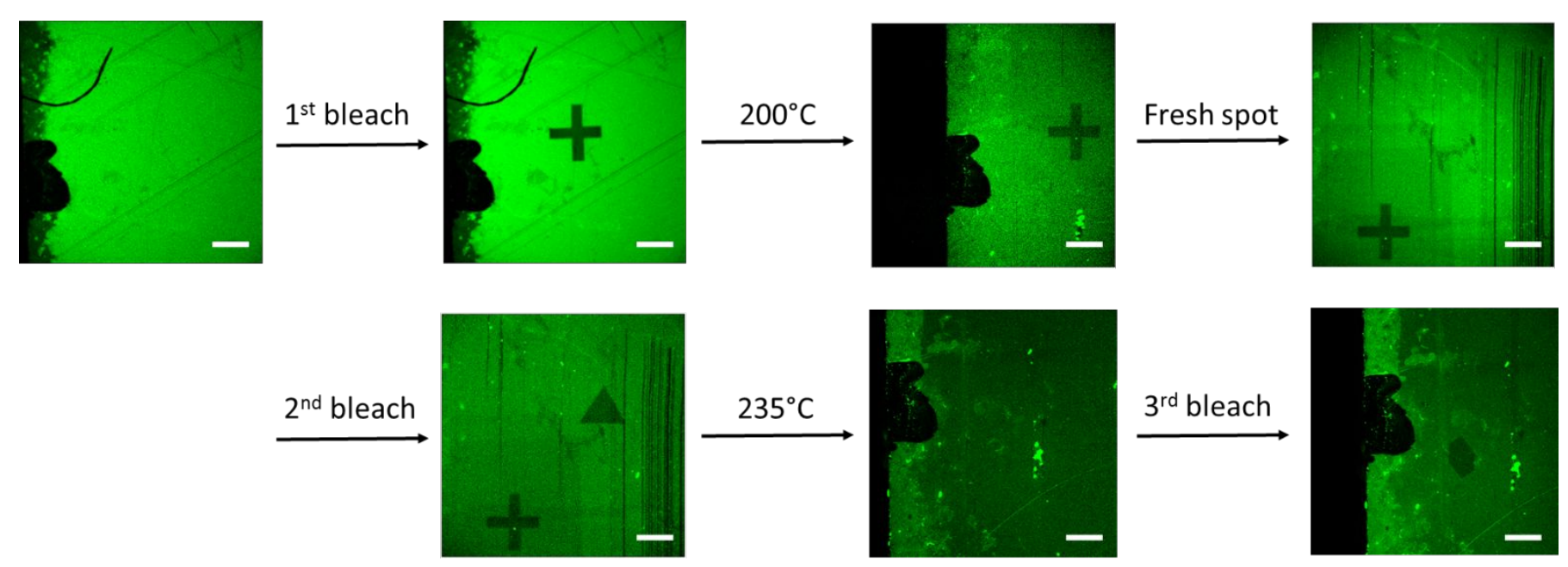

Figure S28. Successful bleaching-regeneration-bleaching cycle by CLSM using the $405 \mathrm{~nm}$ laser on a monomer monolayer transferred on a $285 \mathrm{~nm} \mathrm{SiO}_{2}$-coated silicon wafer (scale bar is $200 \mu \mathrm{m}$ ). The curvy feature in the first two micrographs is a tiny hair contamination. In the first step a cross is bleached (polymerized) on the monolayer, subsequently the wafer is treated at $200^{\circ} \mathrm{C}$ under nitrogen for $2 \mathrm{~h}$ in order to trigger the [4+4]-retro cycloaddition (depolymerization) and recover the fluorescence. Unfortunately the heating process drastically degrades the monolayer with overall fluorescence intensity losses exceeding $60 \%$. The monolayer can nevertheless be bleached again with a triangular shape on a fresh spot. A second thermal treatment at $235^{\circ} \mathrm{C}$ for $4 \mathrm{~h}$ under nitrogen recovers the fluorescence from the bleached cross, but the overall fluorescence intensity dropped by $80 \%$ from its initial value. A final hexagonal shape can again be bleached on the thermally treated monolayer. After the thermal treatment the sensitivity of the detector had to be increased due to the fluorescence loss. Different times and temperatures were tried for the regeneration process but in all cases, fluorescence loss was observed indicating degradation of the monolayer. As a reference, for the hydrocarbon anthraphane the [4+4]-retrocycloaddition could be induced at $180^{\circ} \mathrm{C}$ in the dimers and at $200^{\circ} \mathrm{C}$ in its linear polymers. ${ }^{10}$ These temperatures are unfortunately too high for monolayers of 1. 

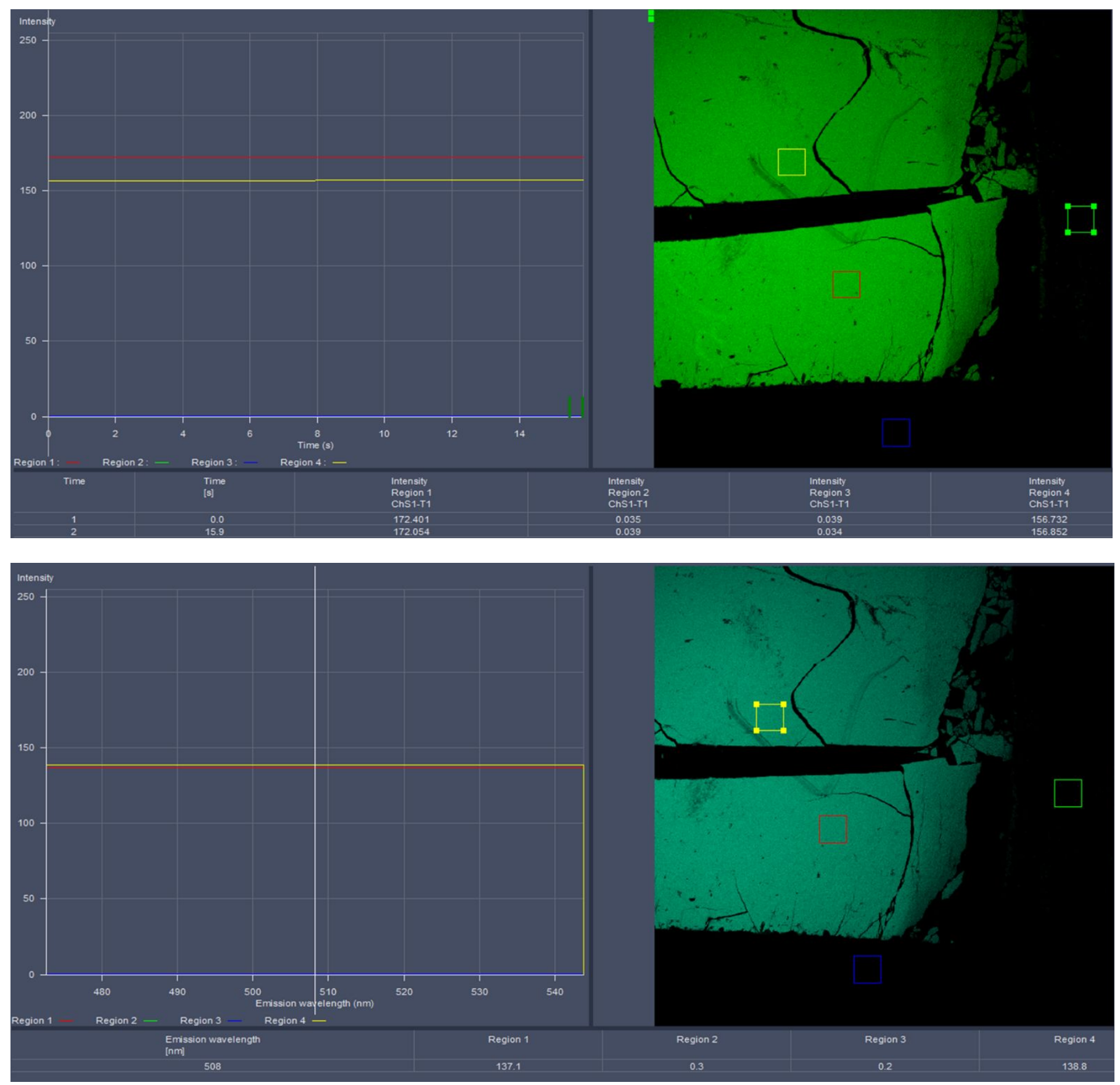

Figure S29. Control experiment of the stability of the monolayer according to fluorescence emission. The top picture is a freshly prepared monolayer while the bottom picture is the same monolayer after being stored for 5 days in a drawer. The same detector settings were used and a fluorescent decrease of approximately $10 \%$ was observed. Nonirradiated regions (red and yellow), control region (green). 


\section{Patterned Lithography in Monolayers}
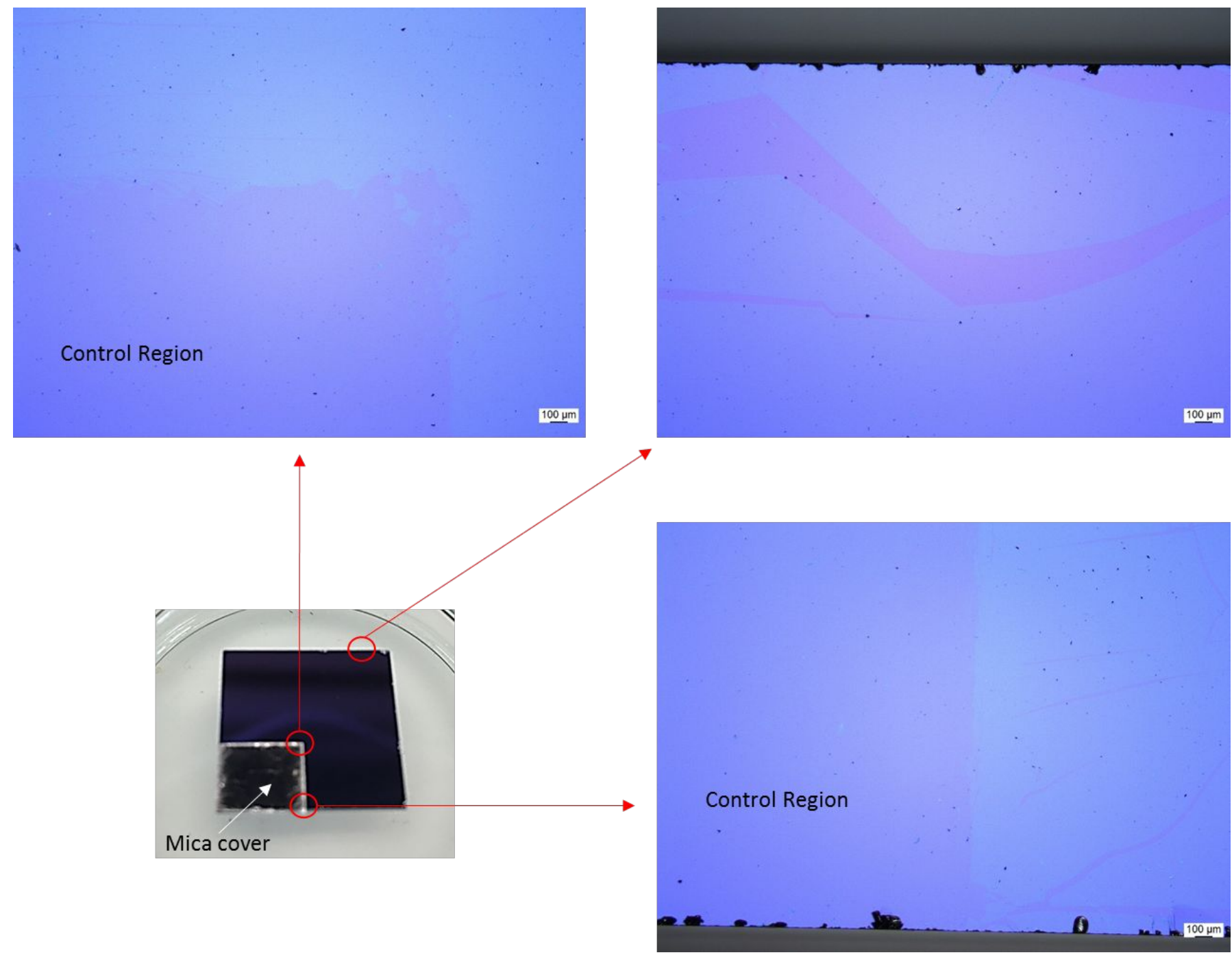

Figure S30. Optical micrographs in differential interference contrast (DIC) mode and the corresponding positions on the silicon oxide wafer. There is a clear difference in contrast between monolayer regions which appear sky blue and bare silicon oxide which appear violet in color. A mica cover was put on the wafer before transferring the monolayer in order to have defined monolayer-free area as control region. After the transfer and drying, the mica is removed. In some places the monolayer is naturally cracked due to the transfer process. 
Before Irradiation
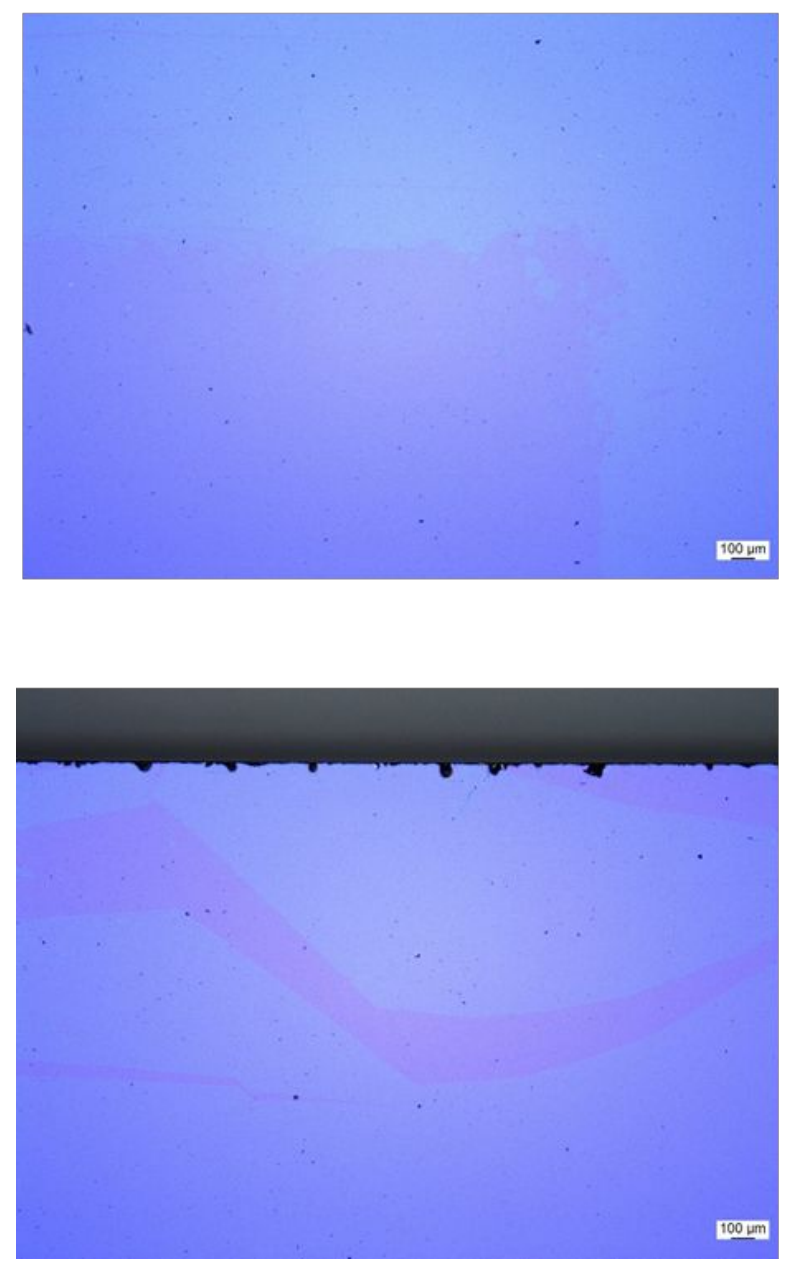

After Irradiation
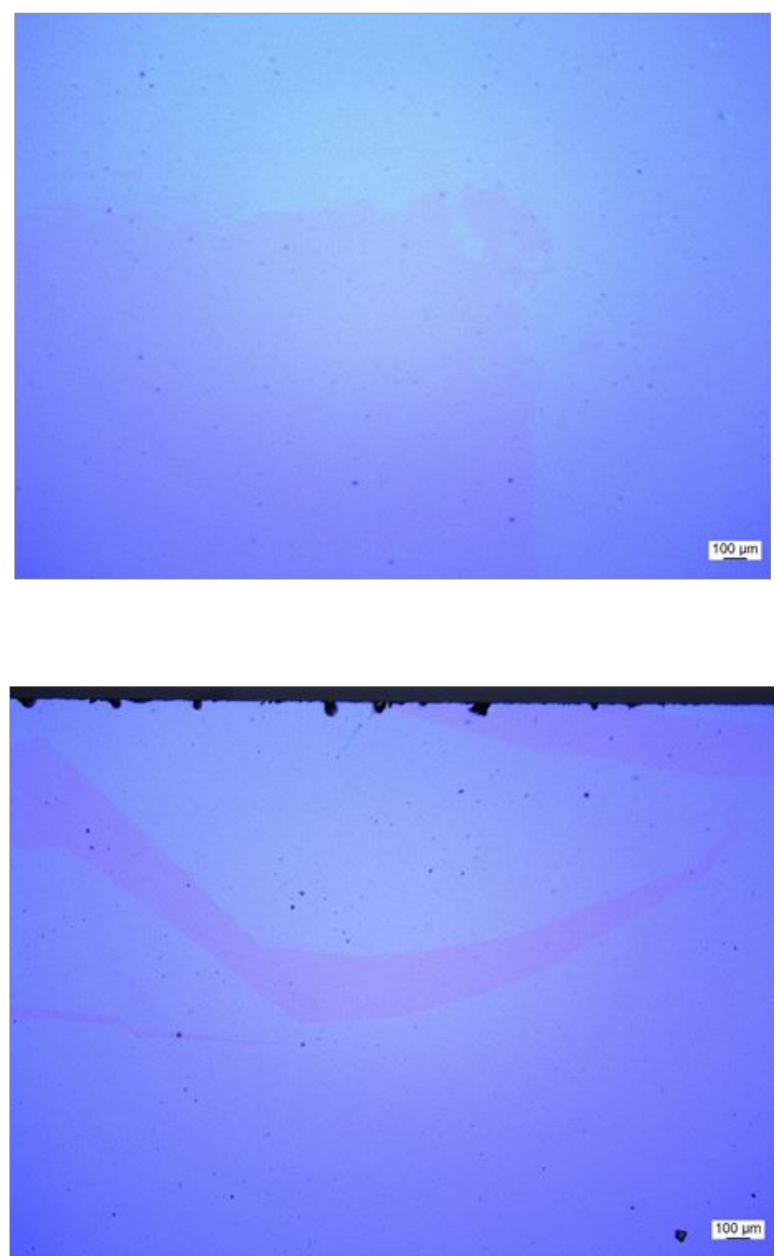

Figure S31. Optical micrographs in differential interference contrast (DIC) of non-irradiated (left) and irradiated monolayer (right). There is no contrast difference and no difference in color arising from the polymerization process. 

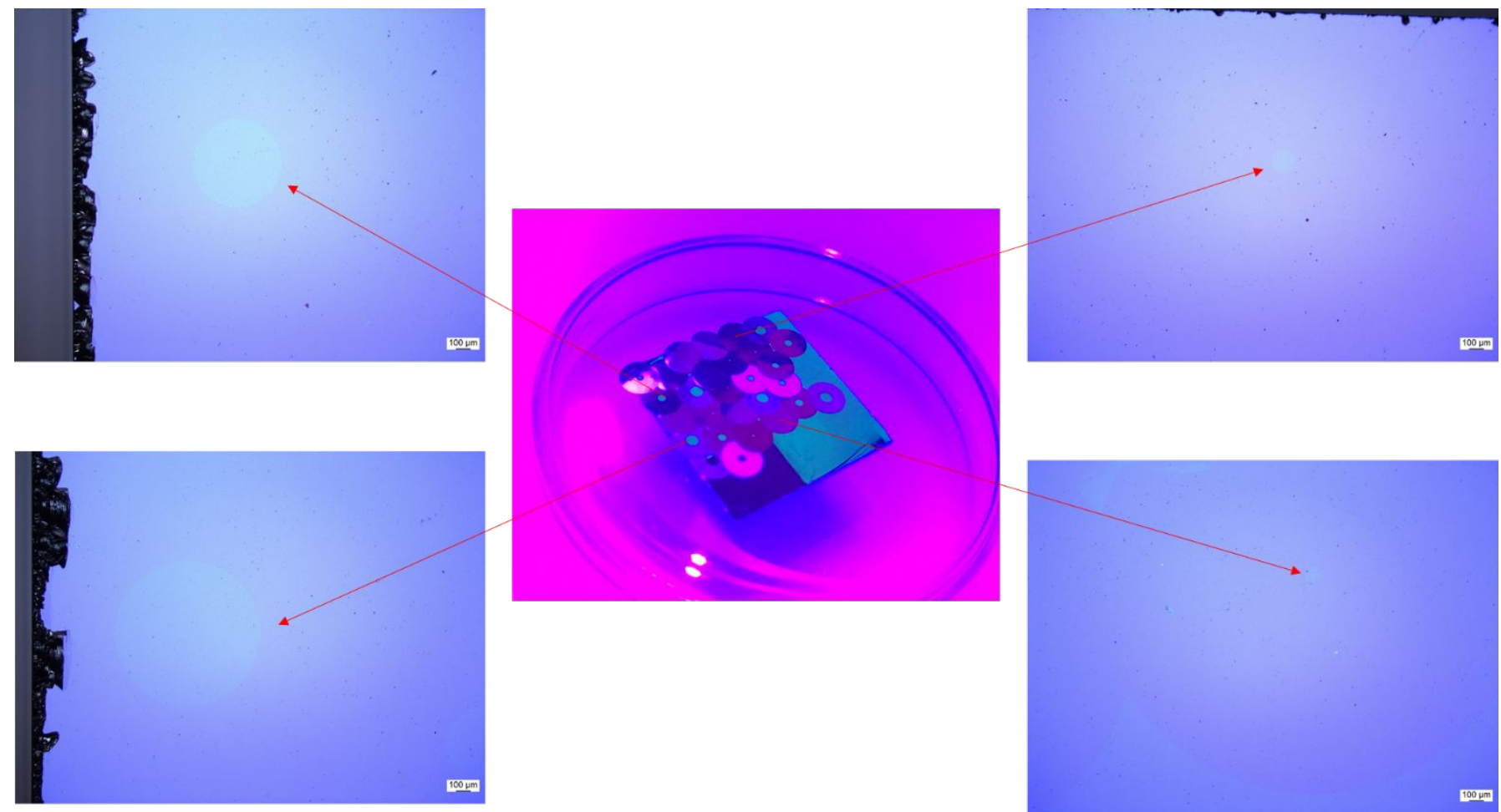

Figure S32. Optical micrographs in differential interference contrast (DIC) of the silicon oxide wafer after the lithographic process with the corresponding positions of the TEM grids. One can observe the circular pattern imprinted on the wafer as a monolayer. The diameter of the circles correspond to the diameters of the single-hole TEM grids used as mask, clockwise from top-left: $600 \mu \mathrm{m}, 200 \mu \mathrm{m}, 100 \mu \mathrm{m}$ and $1000 \mu \mathrm{m}$. The monolayer regions appear sky blue whereas the bare silicon oxide is violet, the same colors observed prior to the polymerization reaction.

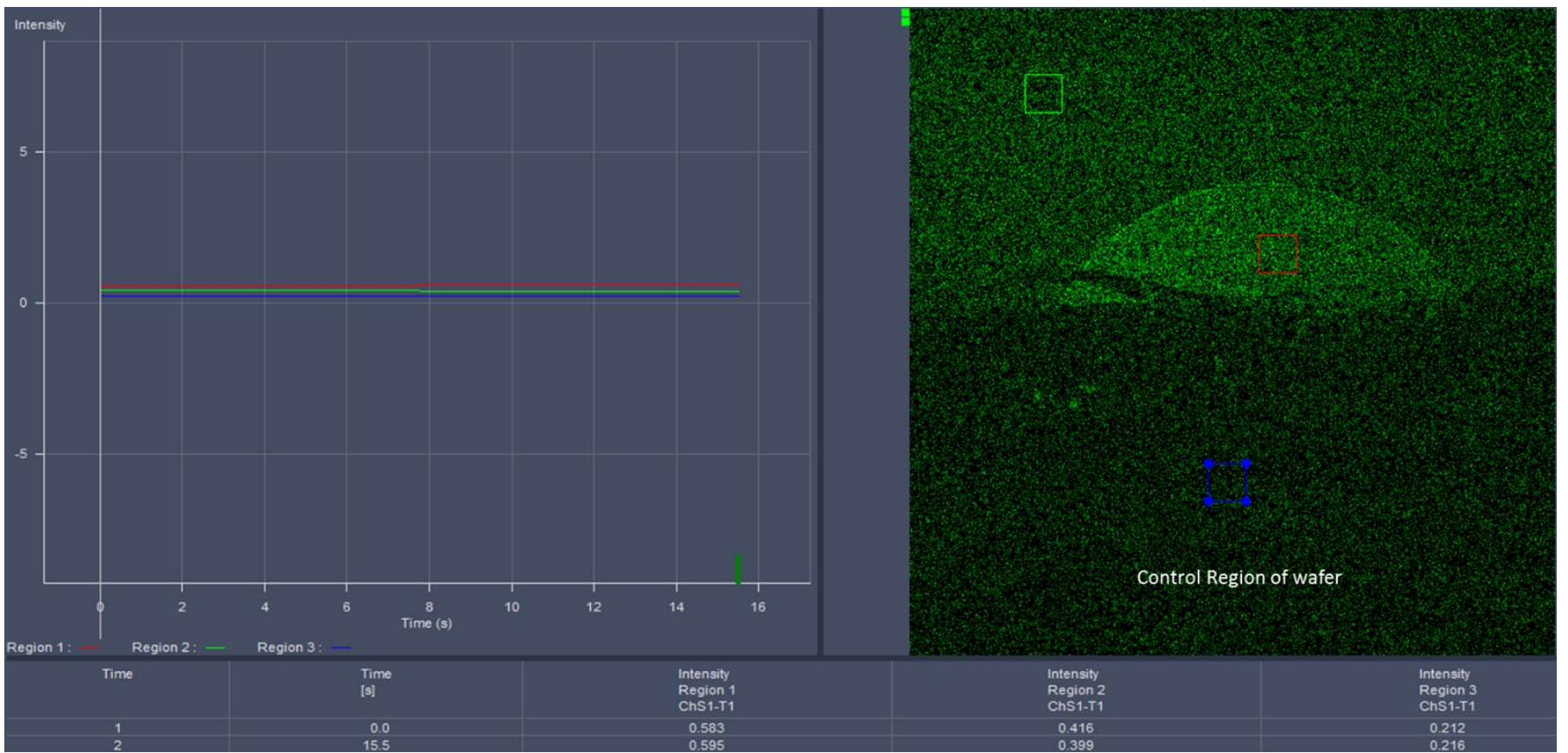

Figure S33. After the lithographic process at increased detector settings in the CLSM, the difference in fluorescence intensities between control, irradiated, and washed regions is minimal. 


\section{Molecular model for monomer and polymer monolayer}

All calculations were performed in the ADF modeling suite using density functional theory (DFT) methods; the cluster model calculations were done in the program $\mathrm{ADF}^{11}$ and periodic model calculations in BAND. ${ }^{12}$ The functional PBE ${ }^{13}$ was used in all calculations together with the D3 empirical dispersion correction ${ }^{14}$ with Becke-Johnson damping. ${ }^{15}$ The TZP basis set was utilized for all cluster model calculations and DZP for calculations with periodic structures. ${ }^{16}$ To simplify the calculations, the monomer structure was slightly altered by shortening the DEGME chains by three atoms, resulting in ethylene glycol monomethyl ether (EGME) chains (Figure S34). Monomer molecules were first fully optimized in gas phase, then the aromatic part of the molecule was frozen and only the ether chains were optimized. Optimizations were performed also in the COSMO implicit solvent model. ${ }^{17,18}$ The monolayer structures were fully optimized with the two-dimensional periodic boundary conditions; the unit cell shape and area were optimized together with atom positions.
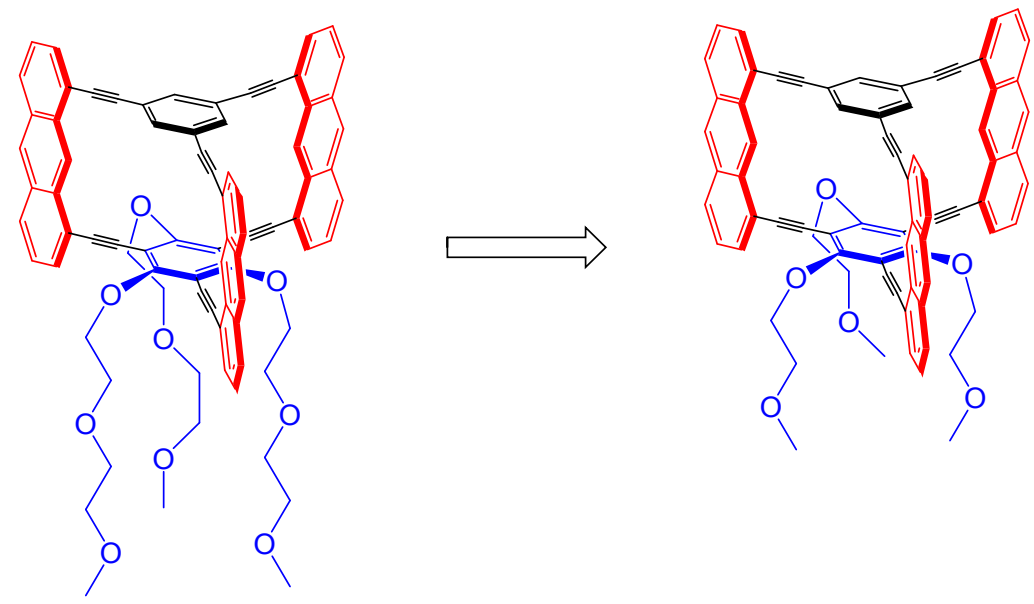

Figure S34. Simplification of the monomer structure by shortening the DEGME chains.

Monomer structures were first optimized in the gas phase and then re-optimized in water solvent, in form of an implicit COSMO solvent. We found six possible conformations of the ether chains (Figure S35). The monomer structure has $C_{3}$ symmetry for conformations $1,2,3,4$ and 6 , whereas for conformation $5, C_{3 v}$ symmetry was observed. Conformations 3 and 6 were found to be more favorable energetically, with 3 being the preferred one. In this conformation the dispersion interaction between anthracenes and ether chains is maximal. After addition of water solvent the energy preference for conformation 3 is even increased. Structurally, there is only a minimal difference between the gas phase and water environment. Therefore, it is sufficient to study the solvation in periodic model only by single point calculations with the gas-phase structures. 

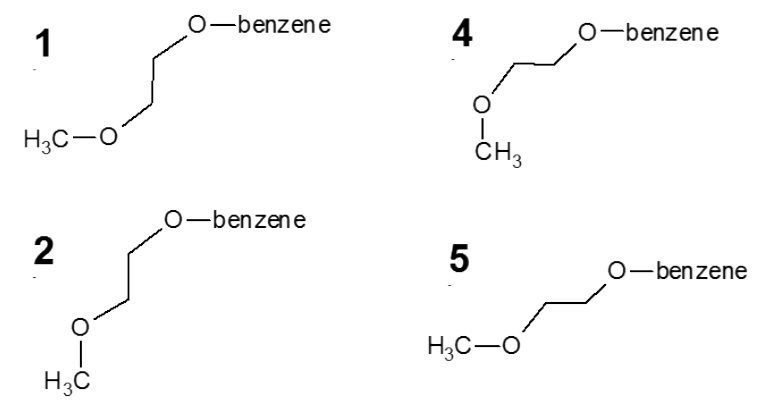

3

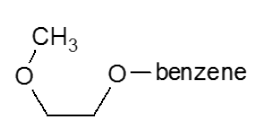

\section{6}

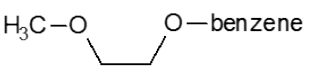

Figure S35. Possible conformations of the EGME ether chains.

Table S2. Relative energies of monomer with different conformations of EGME chains in gas phase and with addition of water as an implicit COSMO solvent calculated at PBE-D3(BJ)/TZP level.

\begin{tabular}{ccc}
\hline \multirow{2}{*}{ structure } & \multicolumn{2}{c}{$\mathrm{E}_{\mathrm{rel}} / \mathrm{kJ} \mathrm{mol}^{-1}$} \\
\cline { 2 - 3 } & Gas Phase & Water (COSMO) \\
\hline $\mathbf{1}$ & 16 & 27 \\
$\mathbf{2}$ & 10 & 24 \\
$\mathbf{3}$ & 0 & 0 \\
$\mathbf{4}$ & 18 & 17 \\
$\mathbf{5}$ & 8 & 24 \\
$\mathbf{6}$ & 6 & 3 \\
\hline
\end{tabular}
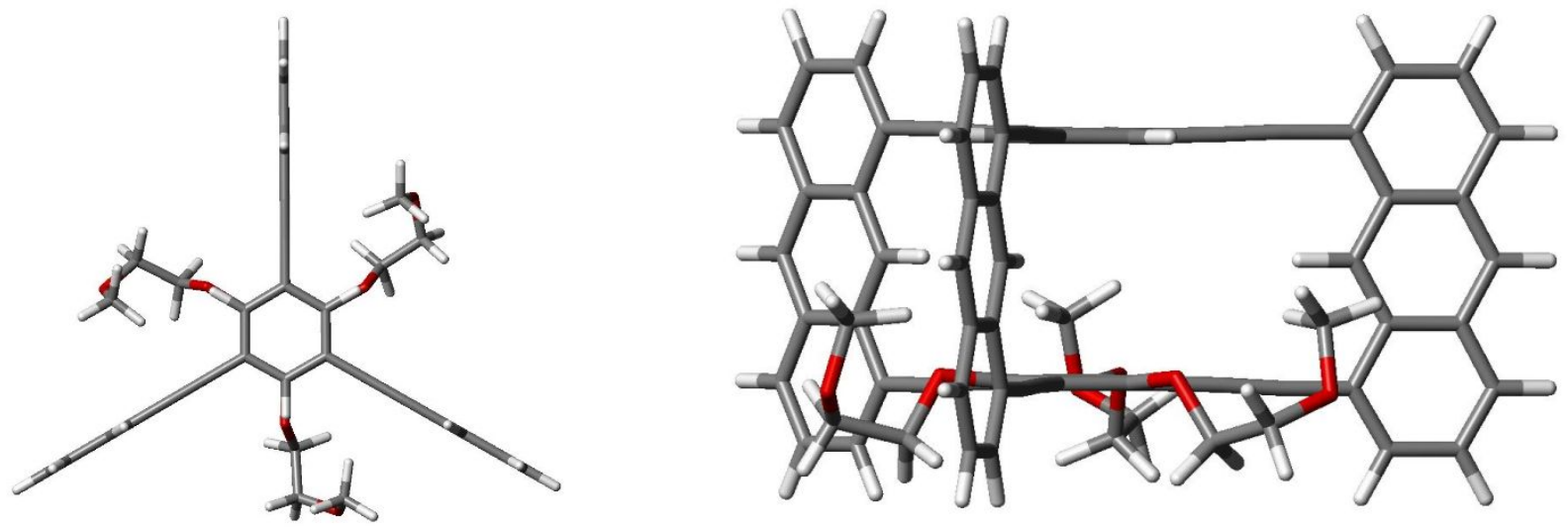

Figure S36. Monomer structure optimized at PBE-D3(BJ)/TZP level with COSMO implicit water solvent. The ether chains are in the conformation 3 , having a $C_{3}$ symmetry. 


\section{Monomer monolayer structure}

For the monomer monolayer at the air/water interface we propose a hexagonal packing with all the anthracene units of 1 stacked face-to-face (Figure S37).

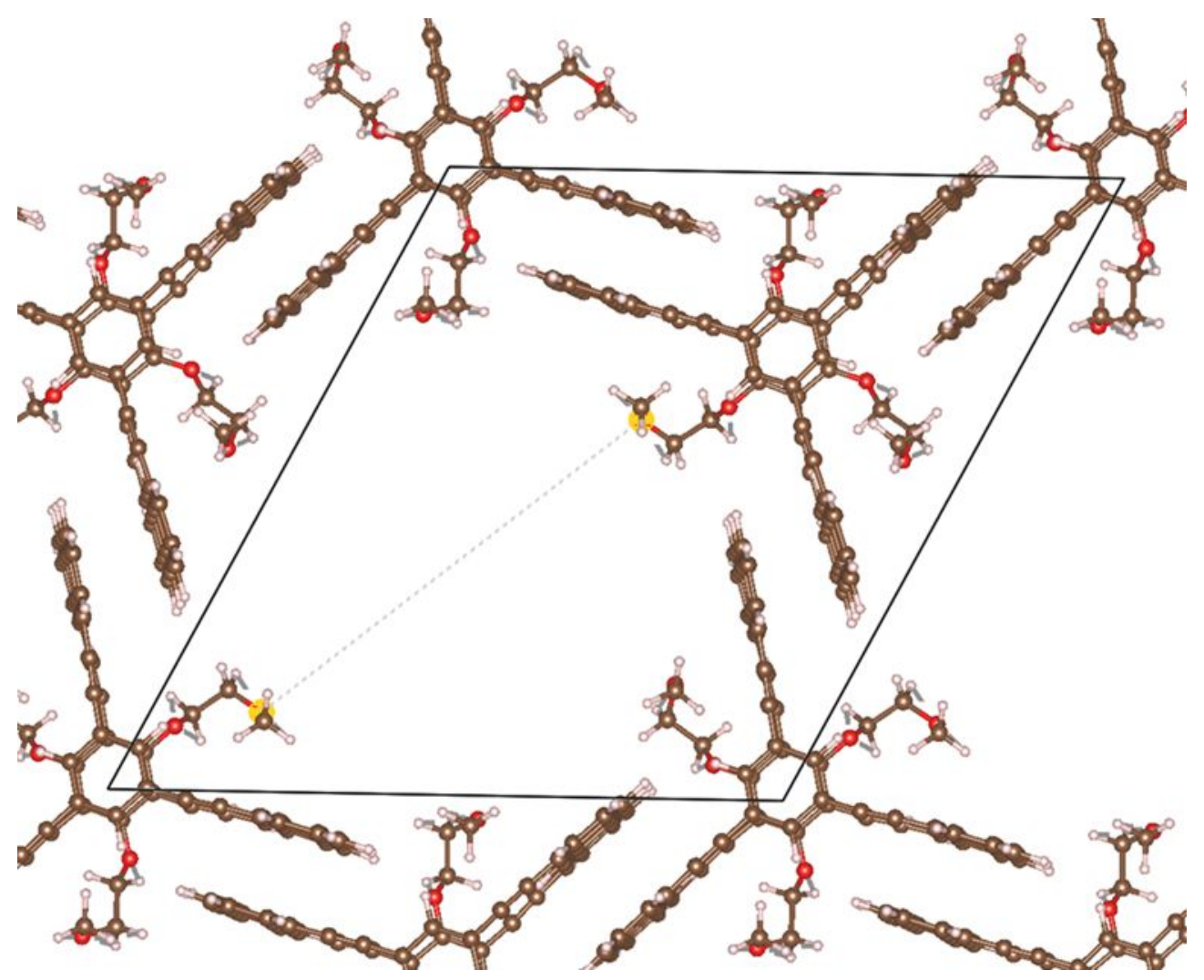

Figure S37. DFT optimized structure of monomer monolayer with the proposed packing viewed from top. With this packing a MMA value of $252 \AA^{2}$ was calculated (from the unit cell parameters, with two monomers per unit cell: $\left.23.26 \AA \times 24.45 \AA \times \sin \left(62.25^{\circ}\right) / 2=252 \AA^{2}\right)$. This value is in good agreement with the measured value for an uncompressed self-assembled monolayer.

The monomer monolayer structure with ether chains conformation 3 is the most stable (Table S4). The lattice parameters are $a=23.264 \AA, b=24.450 \AA, \gamma=62.25^{\circ}$. The structure is porous with a pore diameter of $13.5 \AA$, calculated from the distance between the two closest $\mathrm{O}$-atoms across the channels, accounting for vdW radii (Figure SX). Note that only the monomer monolayers 3, 5 and 6 were fully optimized, due to very high relative energies of the others in both isolated monomer and polymer structures and very slow convergence of the structure optimizations in case of monolayer.

The monomers in the structure are slightly tilted to allow the anthracene to have an optimal interaction with each other; in the experimental structure every monomer can be tilted differently due to conformational entropy, resulting in an uneven surface structure. 


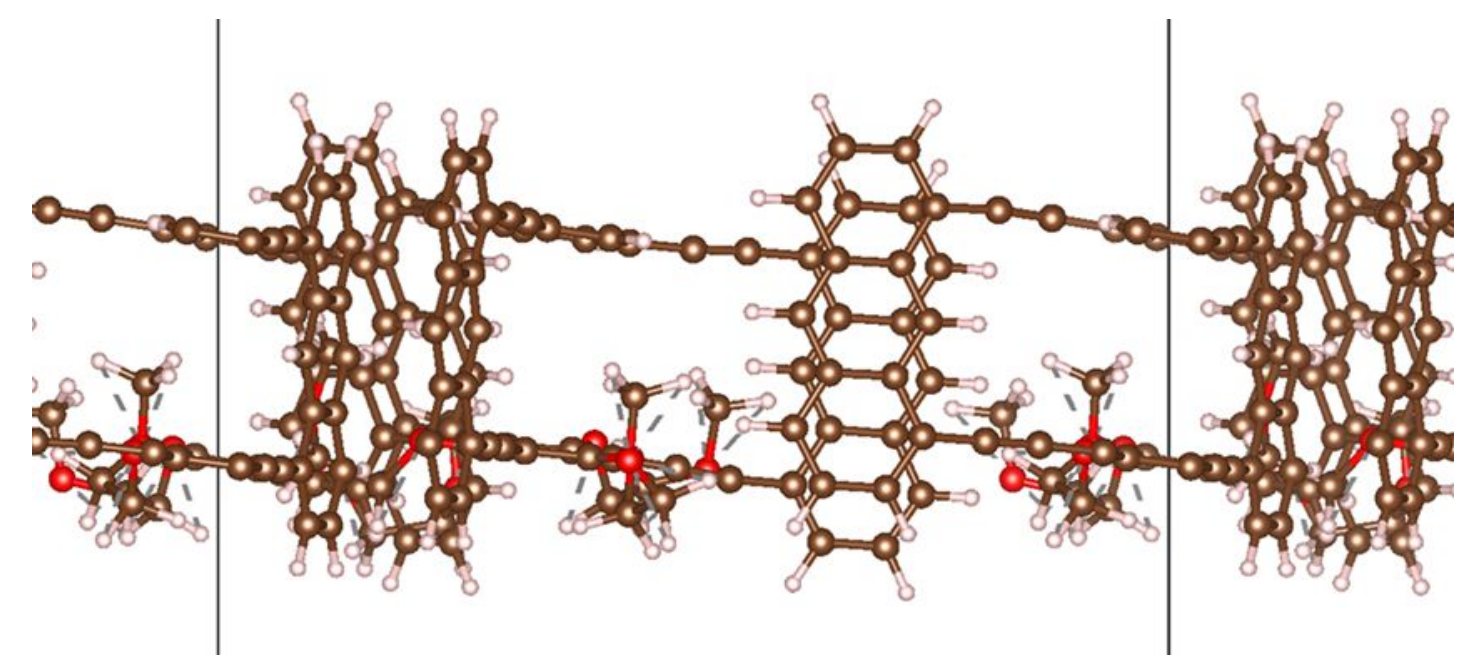

Figure S38. Calculated monomer monolayer packing seen along the the $b$-axis.

We also calculated a monomer packing in which the anthracene units are all in an edge-to-face relationship: this packing motif is commonly found in anthraphane single crystals. To compare the packing we have optimized the monomer monolayer structures with ether chains shortened to only hydroxyl groups (Figure S39). This eliminates the effect of the chain on energetics of the structure and allows us to study only the effect of the packing. The faceto-face packing was found to be more stable by $18.6 \mathrm{kcal} / \mathrm{mol}$ per unit cell, i.e. $9.3 \mathrm{kcal} / \mathrm{mol}$ per monomer (Table S3).

\section{ftf-packing}

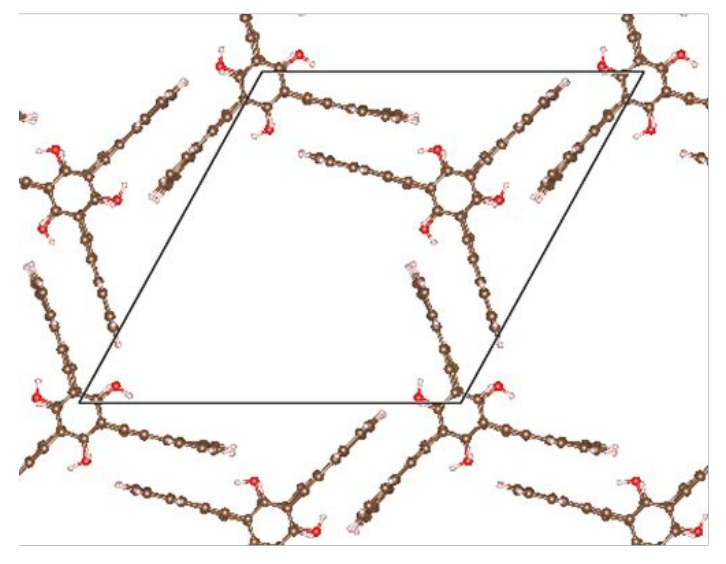

etf-packing

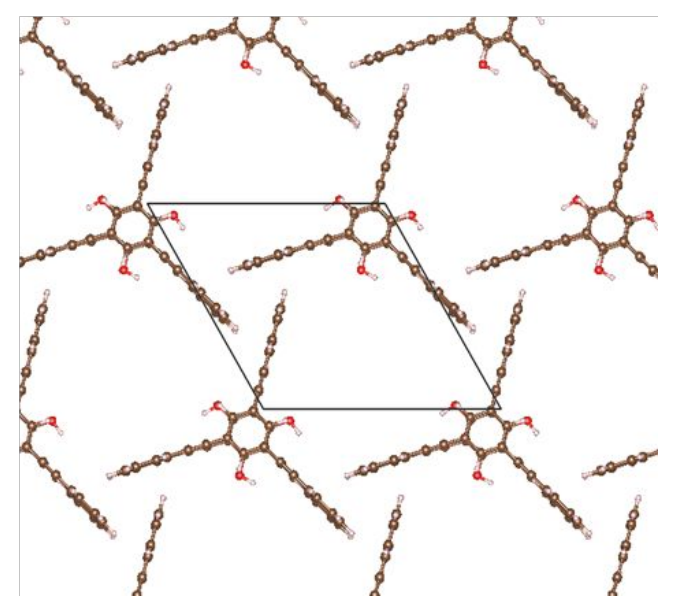

Figure S39. All-ftf and all-etf monomer monolayer packings in the gas phase, as seen from top. Structures were optimized using PBE-D3(BJ)/DZP method. 
Table S3. Relative energies for the all-etf and all-ftf monomer monolayer packings in comparison, obtained at PBED3(BJ)/DZP level. For both packing models, the chains were shortened to hydroxyl groups.

\begin{tabular}{cc}
\hline structure & $\begin{array}{c}\mathrm{E}_{\mathrm{rel}} / \mathrm{kJ} \mathrm{mol}^{-1} \text { per UC } \\
(2 \text { monomers per UC })\end{array}$ \\
\cline { 2 - 2 } & Gas Phase \\
\hline $\begin{array}{c}f t f \\
\text { etf }\end{array}$ & 0 \\
(double UC) & 78 \\
\hline
\end{tabular}

\section{Polymer monolayer structure}

The polymer structure with ether chains conformation 3 was found to be the optimal one for the polymer structure, similarly to the monomer monolayer (Table S4). Lattice parameters: $a=24.455 \AA, b=24.670 \AA, \gamma=119.27^{\circ}$. The pore diameter is $14.3 \AA$, calculated from the distance between the two closest O-atoms across the channels, accounting for vdW radii.

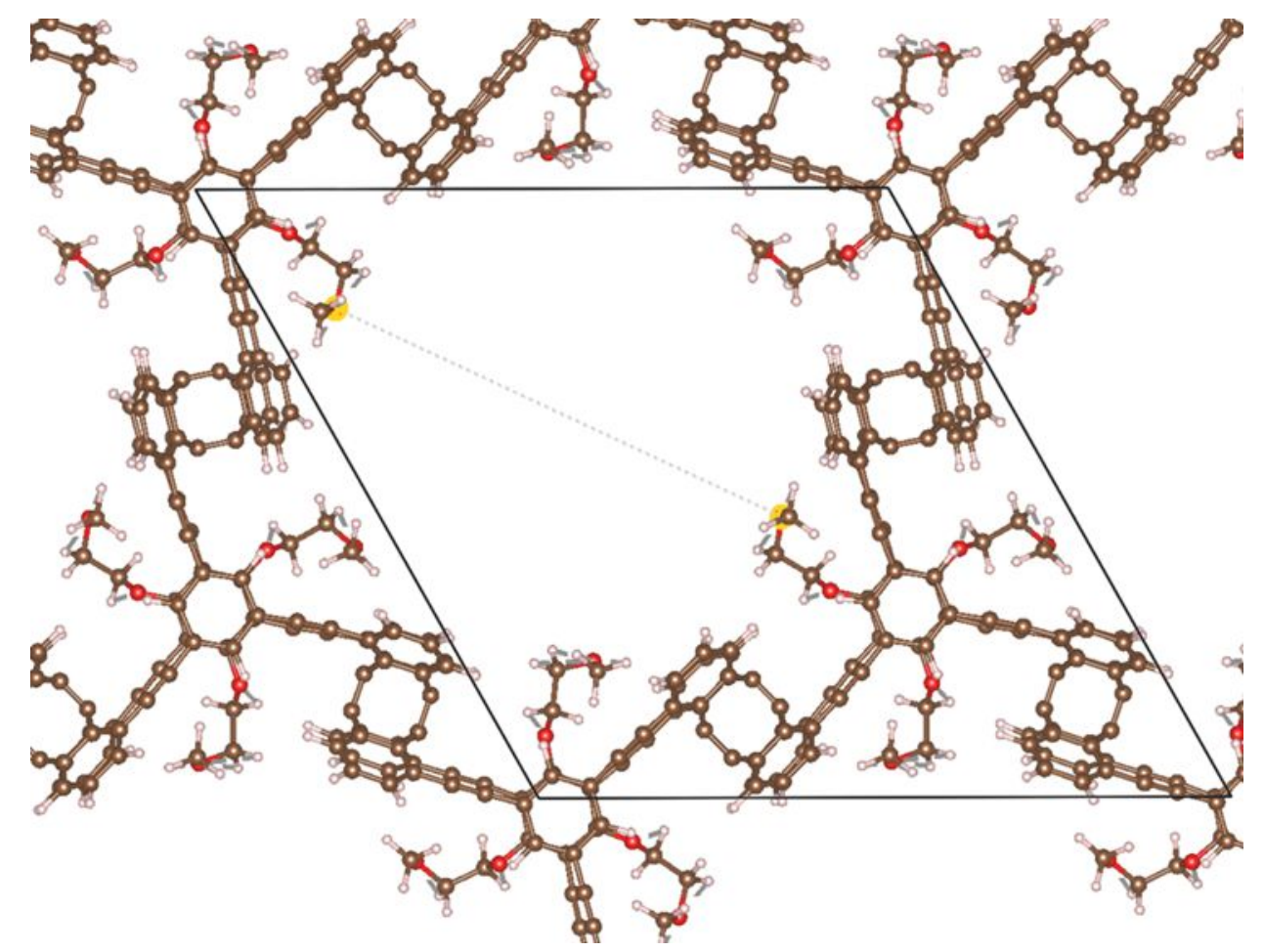

Figure S40. Polymer structure optimized using PBE-D3(BJ)/DZP method, view from top. 


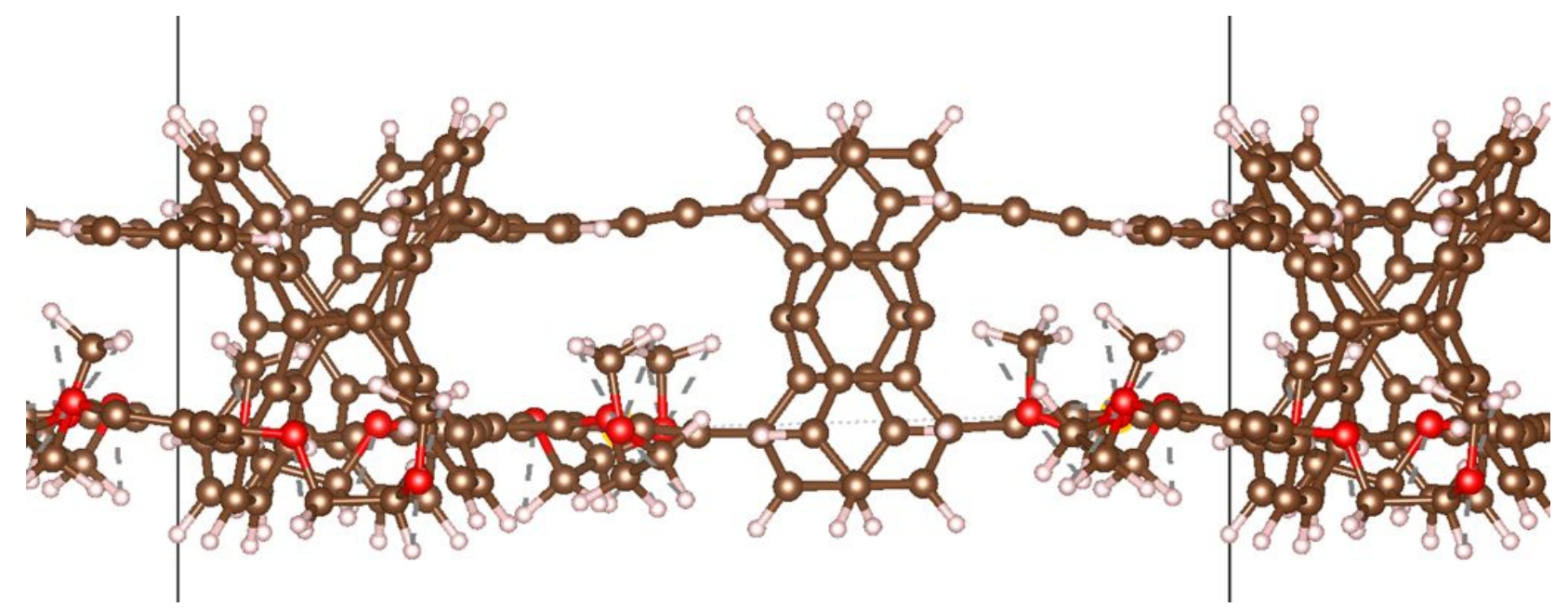

Figure S41. Polymer structure, optimized using PBE-D3(BJ)/DZP method, view along the $b$-axis.

Table S4. Relative energies selected monomer monolayer structures and polymer with different ether chains conformations in gas phase and with addition of water as implicit COSMO solvent, calculated at PBE-D3(BJ)/DZP level. Computationally demanding optimizations of monomer monolayer in conformations 1,2 and 4 were not pursued due to very large relative energies. Conformation 3 is the most stable in both polymer and monomer monolayer.

\begin{tabular}{ccccc}
\hline & \multicolumn{4}{c}{$\begin{array}{c}\mathrm{E}_{\mathrm{rel}} / \mathrm{kJ} \mathrm{mol}^{-1} \text { per UC } \\
(2 \text { monomers per UC) }\end{array}$} \\
\cline { 2 - 5 } structure & \multicolumn{4}{c}{ Polymer } \\
\cline { 2 - 5 } & Monomer monolayer & Gas Phase & Water (COSMO) \\
\cline { 2 - 5 } $\mathbf{1}$ & Gater (COSMO) & 122 & 128 \\
$\mathbf{2}$ & & 102 & 105 \\
$\mathbf{3}$ & 0 & 0 & $\mathbf{0}$ & $\mathbf{0}$ \\
$\mathbf{4}$ & & & 80 & 108 \\
$\mathbf{5}$ & 13 & & 48 & 95 \\
$\mathbf{6}$ & 1 & 30 & 30 & 49 \\
\hline
\end{tabular}




\section{Amphiphilicity and spreading of anthraphanes at the air/water inteface}

The interfacial behavior of anthraphane-tri(OMe) $\mathbf{2}^{19}$ was also tested by spreading a dilute solution of the monomer in chloroform $(0.25 \mathrm{mg} / \mathrm{mL})$ at the air/water interface. SP vs MMA isotherm was recorded and film formation was followed by BAM. The results are summarized in Figures S42 and S43. Just by looking at the MMA, one sees immediately that the values are far below the expected ones: the curve slowly increases until $50 \AA^{2}$ and then steeply grows until a SP of $60 \mathrm{mN} / \mathrm{m}$ is reached. Such MMA values are typical for molecules with a very small cross-section such as fully stretched aliphatic chain amphiphiles ${ }^{20}$ (i.e. decanoic acid) and are incompatible with the structure of 2. Such small MMAs are an indication of an inhomogeneous distribution of thickness due to low amphiphilicity: the molecules lack a preferred orientation upon compression and therefore continuously slide on top of each another without a visible transition.
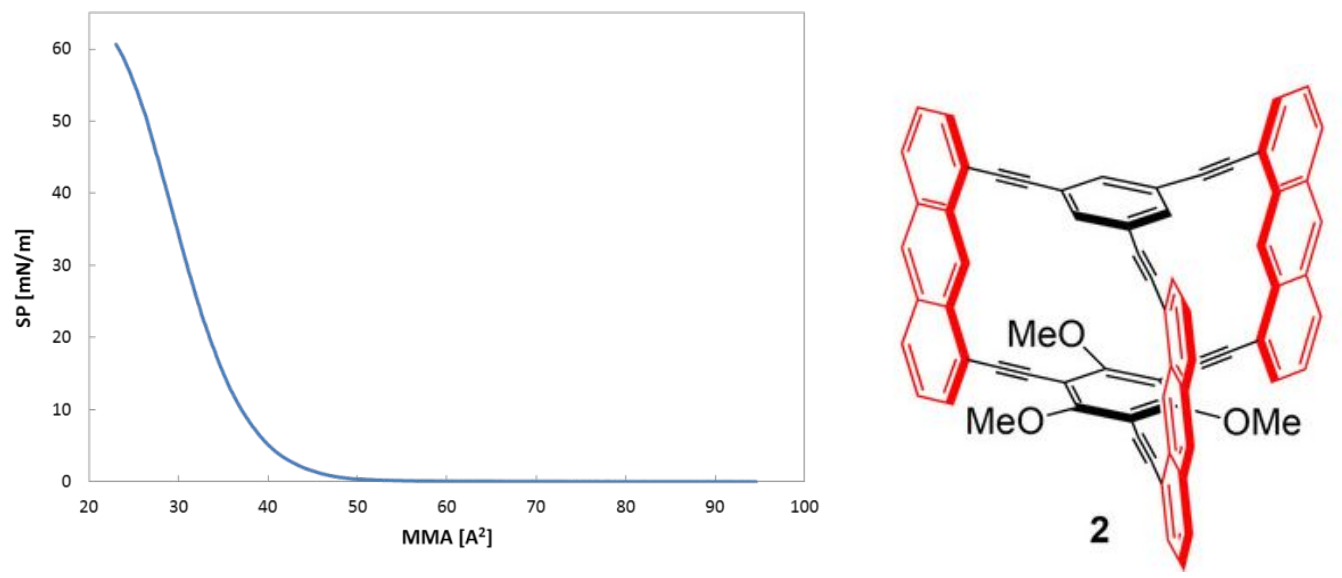

Figure S42. SP vs MMA isotherm of anthraphane $\mathbf{2}$ at the air/water interface. The low MMA values indicate multilayer formation, which could be a consequence of the low amphiphilicity of the molecule.

Confirmation of such inhomogeneous thickness distribution came from BAM analysis (Figure S42): the film always appeared inhomogeneous, with thicker domains clearly visible due to the higher contrast. The clear defined edges of the domains observed in the micrographs would suggest a crystallization of the molecule on top of the surface of water. 

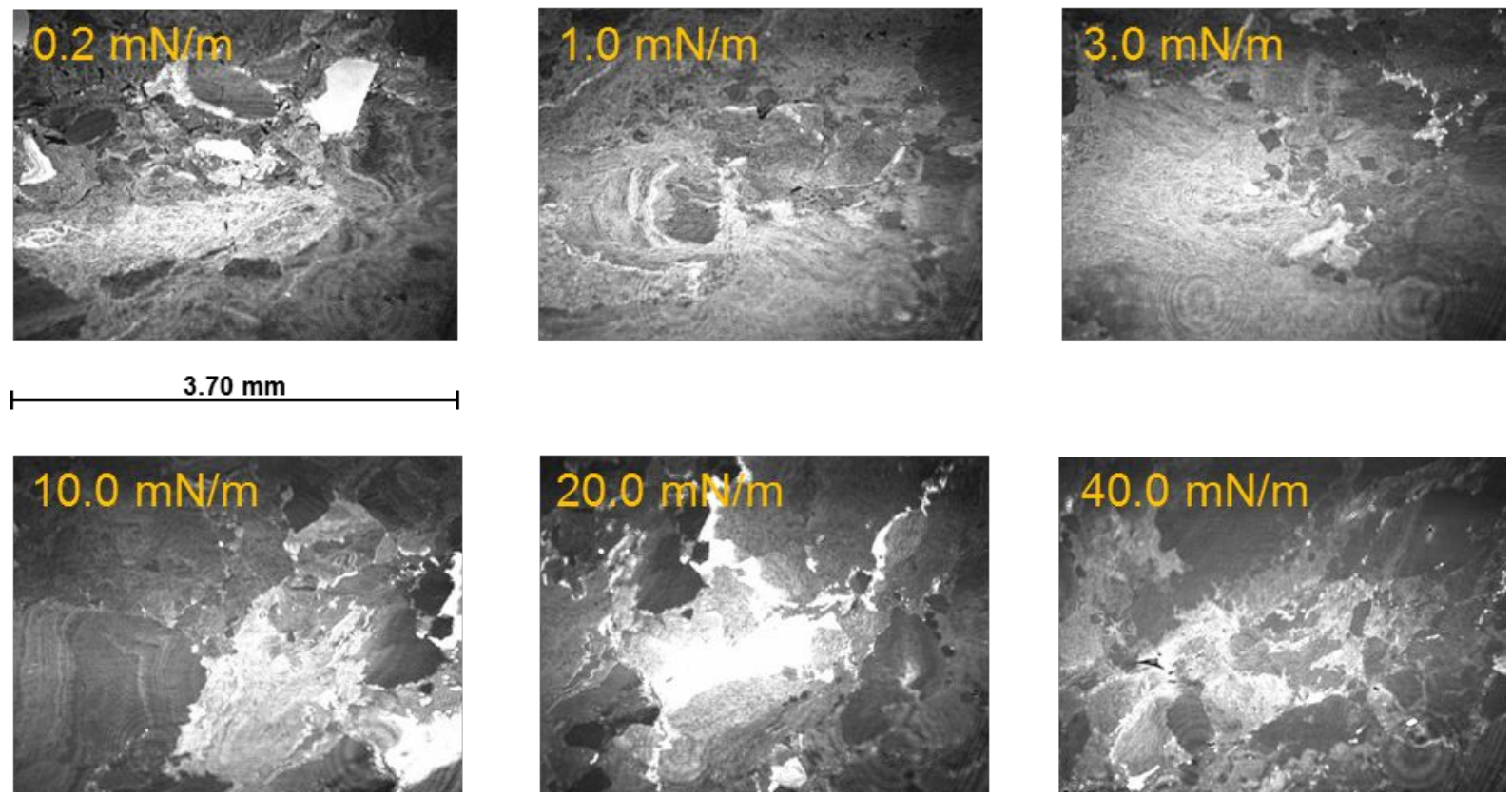

Figure S43. BAM micrographs recorded at different surface pressures during the compression process. The film always looks very inhomogeneous independently from the surface pressure: thicker domains are clearly visible (higher contrast) and confirm multilayer formation.

From these results it is clear that the methoxy groups on the benzene core do not confer sufficient amphiphilicity and that more amphiphilic anthraphanes such as $\mathbf{1}$ are necessary in order to cleanly spread at the air/water interface and form monolayers. 


\section{References}

[1] Williams, D. B. G; Lawton, M. J. Drying of Organic Solvents: Quantitative Evaluation of the Efficiency of Several Desiccants. Org. Chem. 2010, 75, 8351-8354.

[2] Coulson, D. R.; Satek, L. C.; Grim, S. O. Tetrakis(triphenylphosphine)palladium(0). Inorg. Synth. 1971, 13, 121124.

[3] Servalli, M.; Trapp, N.; Wörle, M.; Klaerner, F.-G. Anthraphane: An Anthracene-Based, Propeller-Shaped $D_{3 h^{-}}$ Symmetric Hydrocarbon Cyclophane and Its Layered Single Crystal Structures. J. Org. Chem. 2016, 81, 25722580 .

[4] Servalli, M.; Trapp, N.; Solar, M.; Schlüter, A. D. Library of Single Crystal Structures of a $D_{3 \mathrm{~h}}$-Symmetric Hydrocarbon Cyclophane: A Comprehensive Packing Study of Anthraphane from 30 Solvents. Cryst. Growth Des. 2017, 17, 3419-3432.

[5] CrysAlisPro and ABSPACK. Rigaku Oxford Diffraction, 2016.

[6] Sheldrick, G. M. A Short History of SHELX. Acta Cryst. 2008, A64, 112-122.

[7] Dolomanov, O. V.; Bourhis, L. J.; Gildea, R. J.; Howard, J. A. K.; Puschmann, H. OLEX2: A Complete Structure Solution, Refinement and Analysis Program. J. Appl. Cryst. 2009, 42, 339-341.

[8] Banner, L. T.; Richter, A.; Pinkhassik, E. Pinhole-Free Large-Grained Atomically Smooth Au(111) Substrates Prepared by Flame-Annealed Template Stripping. Surf. Interface Anal. 2009, 41, 49-55.

[9] Opilik, L.; Payamyar, P.; Szczerbinsk, J.; Schutz, A. P.; Servalli, M.; Hungerland, T.; Schluter, A. D.; Zenobi, R. Minimally Invasive Characterization of Covalent Monolayer Sheets Using Tip-Enhanced Raman Spectroscopy. ACS Nano 2015, 9 , 4252-4259.

[10] Servalli, M.; Solar, M.; Trapp, N.; Schlüter, A. D. Photochemical Single-Crystal-to-Single-Crystal (SCSC) Reactions of Anthraphane to Dianthraphane and Poly ${ }_{10}$ anthraphane. Cryst. Growth Des. 2017, 17, 65106522.

[11] te Velde, G.; Bickelhaupt, F. M.; Baerends, E. J.; Fonseca Guerra, C.; van Gisbergen, S. J. A.; Snijders, J. G.; Ziegler, T. Chemistry with ADF. J. Comput. Chem. 2001, 22, 931-967.

[12] te Velde, G.; Baerends, E. J. Precise Density-Functional Method for Periodic Structures. Phys. Rev. B 1991, 44, 7888-7903.

[13] Perdew, J. P.; Burke, K.; Emzerhof, M. Generalized Gradient Approximation Made Simple. Phys. Rev. Lett. 1996, 77, 3865-3868.

[14] Grimme, S. Semiempirical GGA-Type Density Functional Constructed with a Long-Range Dispersion Correction. J. Comput. Chem. 2006, 27, 1787-1799.

[15] Grimme, S.; Ehrlich, S.; Goerigk, L. Effect of the Damping Function in Dispersion Corrected Density Functional Theory. J. Comp. Chem. 2011, 32, 1456-1465.

[16] van Lenthe, E.; Baerends, E. Optimized Slater-Type Basis Sets for the Elements 1-118. J. Comp. Chem. 2003, $24,1142-1156$. 
[17] Klamt, A.; Schüürmann, G. COSMO: A New Approach to Dielectric Screening in Solvents with Explicit Expressions for the Screening Energy and Its Gradient. J. Chem. Soc., Perkin Trans. 2 1993, 799-805.

[18] Pye, C. C.; Ziegler, T. An Implementation of the Conductor-Like Screening Model of Solvation within the Amsterdam Density Functional Package. Theor. Chem. Acc. 1999, 101, 396-408.

[19] Servalli, M.; Trapp, N.; Schlüter, A. D. Single-Crystal-to-Single-Crystal (SCSC) Linear Polymerization of a Desymmetrized Anthraphane. Chem. Eur. J. 2018, 24, 15003-15012.

[20] Langmuir, I. The Mechanism of the Surface Phenomena of Flotation. Trans. Faraday Soc. 1920, 15, 62-74. 